\title{
Erratum to: Measurement of the charge asymmetry in top-quark pair production in the lepton-plus-jets final state in pp collision data at $\sqrt{s}=8 \mathrm{TeV}$ with the ATLAS detector
}

\section{ATLAS Collaboration *}

CERN, 1211 Geneva 23, Switzerland

Received: 10 July 2017 / Accepted: 18 July 2017 / Published online: 20 August 2017

(C) The Author(s) 2017. This article is an open access publication

Erratum to: Eur. Phys. J. C (2016) 76:87

DOI 10.1140/epjc/s10052-016-3910-6

In the original paper, Fig. 4 contains the wrong label preliminary. The label has been fixed, while none of the results have changed.

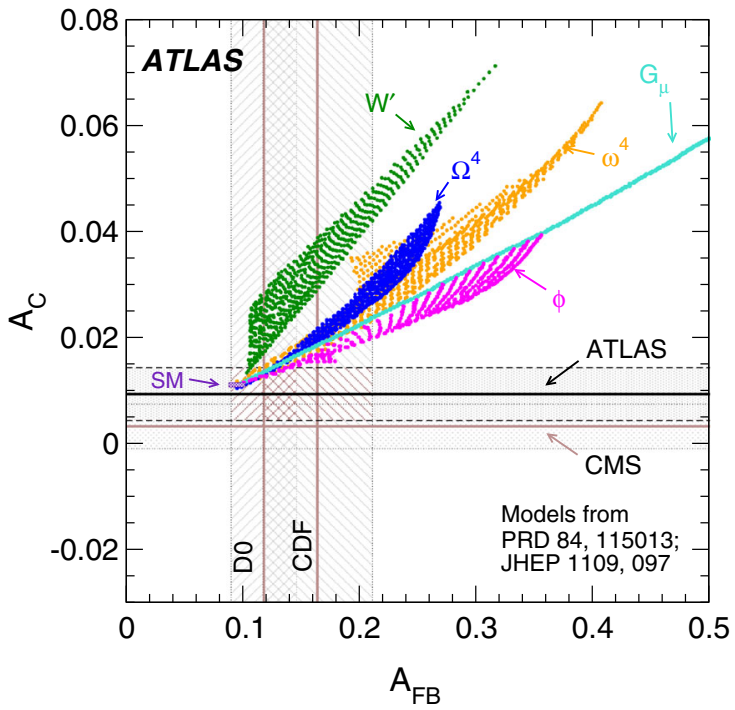

Fig. 4 Measured inclusive charge asymmetries $A_{C}$ at the LHC versus forward-backward asymmetries $A_{\mathrm{FB}}$ at Tevatron, compared with the SM predictions $[1,2]$ as well as predictions incorporating various potential BSM contributions [3,4]: a $W^{\prime}$ boson, a heavy axigluon $\left(\mathcal{G}_{\mu}\right)$, a scalar isodoublet $(\phi)$, a colour-triplet scalar $\left(\omega^{4}\right)$, and a colour-sextet

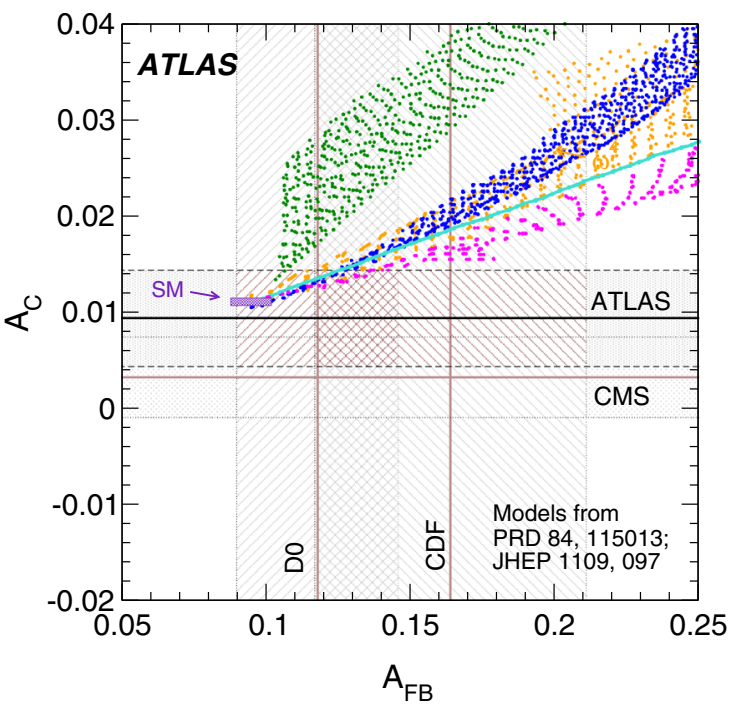

scalar $\left(\Omega^{4}\right)$. The horizontal bands and lines correspond to the ATLAS and CMS measurements, while the vertical ones correspond to the CDF and D0 measurements. The uncertainty bands correspond to a $68 \%$ confidence level interval. The figure on the right is a zoomed-in version of the figure on the left

The online version of the original article can be found under doi:10.1140/epjc/s10052-016-3910-6.

ae-mails: atlas.publications@ @ern.ch 
Open Access This article is distributed under the terms of the Creative Commons Attribution 4.0 International License (http://creativecomm ons.org/licenses/by/4.0/), which permits unrestricted use, distribution, and reproduction in any medium, provided you give appropriate credit to the original author(s) and the source, provide a link to the Creative Commons license, and indicate if changes were made.

Funded by SCOAP ${ }^{3}$.

\section{References}

1. M. Czakon, P. Fiedler, A. Mitov, Resolving the Tevatron top quark forward-backward asymmetry puzzle: fully differential next-tonext-to-leading-order calculation. Phys. Rev. Lett. 115, 052001 (2015). doi:10.1103/PhysRevLett.115.052001. arXiv:1411.3007 [hep-ph]

2. W. Bernreuther, Z.-G. Si, Top quark and leptonic charge asymmetries for the Tevatron and LHC. Phys. Rev. D 86, 034026 (2012). doi:10.1103/PhysRevD.86.034026. arXiv:1205.6580 [hep-ph]

3. J.A. Aguilar-Saavedra, M. Pérez-Victoria, Asymmetries in $t \bar{t}$ production: LHC versus Tevatron. Phys. Rev. D 84, 115013 (2011). doi:10.1103/PhysRevD.84.115013. arXiv:1105.4606 [hep-ph]

4. J.A. Aguilar-Saavedra, M. Pérez-Victoria, Simple models for the top asymmetry: constraints and predictions. JHEP 09, 097 (2011). doi:10.1007/JHEP09(2011)097. arXiv:1107.0841 [hep-ph] 


\section{ATLAS Collaboration}

G. Aad ${ }^{85}$, B. Abbott ${ }^{113}$, J. Abdallah ${ }^{151}$, O. Abdinov ${ }^{11}$, R. Aben ${ }^{107}$, M. Abolins ${ }^{90}$, O. S. AbouZeid ${ }^{158}$, H. Abramowicz ${ }^{153}$, H. Abreu ${ }^{152}$, R. Abreu ${ }^{116}$, Y. Abulaiti ${ }^{146 a, 146 b}$, B. S. Acharya ${ }^{164 a, 164 b, a}$, L. Adamczyk ${ }^{38 a}$, D. L. Adams ${ }^{25}$, J. Adelman ${ }^{108}$, S. Adomeit ${ }^{100}$, T. Adye ${ }^{131}$, A. A. Affolder ${ }^{74}$, T. Agatonovic-Jovin ${ }^{13}$, J. Agricola ${ }^{54}$, J. A. Aguilar-Saavedra ${ }^{126 a, 126 f}$,

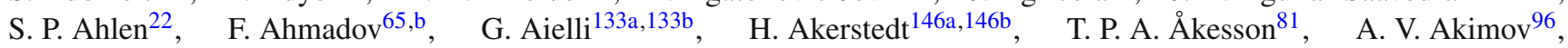

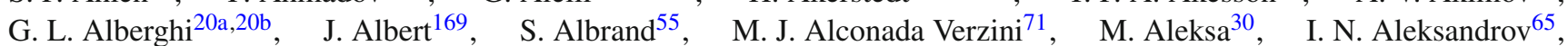

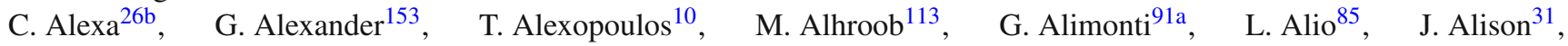
S. P. Alkire ${ }^{35}$, B. M. M. Allbrooke ${ }^{149}$, P. P. Allport ${ }^{18}$, A. Aloisio ${ }^{104 a, 104 b}$, A. Alonso ${ }^{36}$, F. Alonso ${ }^{71}$, C. Alpigiani ${ }^{138}$, A. Altheimer ${ }^{35}$, B. Alvarez Gonzalez ${ }^{30}$, D. Álvarez Piqueras ${ }^{167}$, M. G. Alviggi 104a,104b, B. T. Amadio ${ }^{15}$, K. Amako ${ }^{66}$, Y. Amaral Coutinho ${ }^{24 a}$, C. Amelung ${ }^{23}$, D. Amidei ${ }^{89}$, S. P. Amor Dos Santos ${ }^{126 a, 126 c}$, A. Amorim ${ }^{126 a, 126 b}$, S. Amoroso ${ }^{48}$,

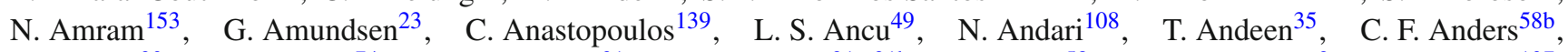
G. Anders $^{30}$, J. K. Anders $^{74}$, K. J. Anderson ${ }^{31}$, A. Andreazza ${ }^{91 a, 91 b}$, V. Andrei ${ }^{58 a}$, S. Angelidakis ${ }^{9}$, I. Angelozzi ${ }^{107}$, P. Anger ${ }^{44}$, A. Angerami ${ }^{35}$, F. Anghinolfi ${ }^{30}$, A. V. Anisenkov ${ }^{109, c}$, N. Anjos ${ }^{12}$, A. Annovi ${ }^{124 a, 124 b}$, M. Antonellii ${ }^{47}$, A. Antonov ${ }^{98}$, J. Antos ${ }^{144 b}$, F. Anulli ${ }^{132 a}$, M. Aoki ${ }^{66}$, L. Aperio Bella ${ }^{18}$, G. Arabidze ${ }^{90}$, Y. Arai ${ }^{66}$, J. P. Araque ${ }^{126 a}$, A. T. H. $\operatorname{Arce}^{45}$, F. A. Arduh ${ }^{71}$, J-F. Arguin ${ }^{95}$, S. Argyropoulos ${ }^{63}$, M. Arik ${ }^{19 a}$, A. J. Armbruster ${ }^{30}$, O. Arnaez ${ }^{30}$, H. Arnold $^{48}$, M. Arratia ${ }^{28}$, O. Arslan ${ }^{21}$, A. Artamonov ${ }^{97}$, G. Artoni ${ }^{23}$, S. Asai ${ }^{155}$, N. Asbah ${ }^{42}$, A. Ashkenazi 153, B. Åsman ${ }^{146 a, 146 b}$, L. Asquith ${ }^{149}$, K. Assamagan ${ }^{25}$, R. Astalos ${ }^{144 a}$, M. Atkinson ${ }^{165}$, N. B. Atlay ${ }^{141}$, K. Augsten ${ }^{128}$, M. Aurousseau ${ }^{145 b}$, G. Avolio $^{30}$, B. Axen ${ }^{15}$, M. K. Ayoub ${ }^{117}$, G. Azuelos ${ }^{95, d}$, M. A. Baak ${ }^{30}$, A. E. Baas ${ }^{58 a}$, M. J. Baca ${ }^{18}$, C. Bacci ${ }^{134 a, 134 b}$, H. Bachacou ${ }^{136}$, K. Bachas ${ }^{154}$, M. Backes ${ }^{30}$, M. Backhaus ${ }^{30}$, P. Bagiacchi ${ }^{132 a, 132 b}$, P. Bagnaia ${ }^{132 a, 132 b}$, Y. Bai ${ }^{33 a}$, T. Bain ${ }^{35}$, J. T. Baines ${ }^{131}$, O. K. Baker ${ }^{176}$, E. M. Baldin ${ }^{109, c}$, P. Balek ${ }^{129}$, T. Balestri ${ }^{148}$, F. Balli ${ }^{84}$, W. K. Balunas ${ }^{122}$, E. Banas $^{39}$, Sw. Banerjee ${ }^{173}$, A. A. E. Bannoura ${ }^{175}$, L. Barak ${ }^{30}$, E. L. Barberio ${ }^{88}$, D. Barberis ${ }^{50 a, 50 b}$, M. Barbero ${ }^{85}$, T. Barillari ${ }^{101}$, M. Barisonzi ${ }^{164 a, 164 b}$, T. Barklow ${ }^{143}$, N. Barlow ${ }^{28}$, S. L. Barnes ${ }^{84}$, B. M. Barnett ${ }^{131}$, R. M. Barnett ${ }^{15}$, Z. Barnovska $^{5}$, A. Baroncelli134a, G. Barone ${ }^{23}$, A. J. Barr ${ }^{120}$, F. Barreiro ${ }^{82}$, J. Barreiro Guimarães da Costa ${ }^{57}$, R. Bartoldus ${ }^{143}$, A. E. Barton ${ }^{72}$, P. Bartos ${ }^{14 a}$, A. Basalaev ${ }^{123}$, A. Bassalat ${ }^{117}$, A. Basye ${ }^{165}$, R. L. Bates ${ }^{53}$, S. J. Batista ${ }^{158}$, J. R. Batley ${ }^{28}$, M. Battaglia ${ }^{137}$, M. Bauce ${ }^{132 a, 132 b}$, F. Bauer ${ }^{136}$, H. S. Bawa ${ }^{143, e}$, J. B. Beacham ${ }^{111}$, M. D. Beattie ${ }^{72}$,

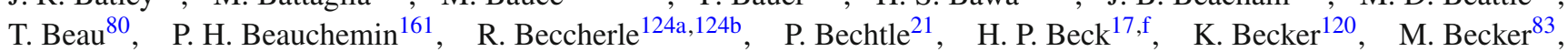
M. Beckingham ${ }^{170}$, C. Becot ${ }^{117}$, A. J. Beddall ${ }^{19 b}$, A. Beddall ${ }^{19 b}$, V. A. Bednyakov ${ }^{65}$, C. P. Bee ${ }^{148}$, L. J. Beemster ${ }^{107}$, T. A. Beermann ${ }^{30}$, M. Begel ${ }^{25}$, J. K. Behr ${ }^{120}$, C. Belanger-Champagne ${ }^{87}$, W. H. Bell ${ }^{49}$, G. Bella ${ }^{153}$, L. Bellagamba ${ }^{20 a}$, A. Bellerive ${ }^{29}$, M. Bellomo ${ }^{86}$, K. Belotskiy ${ }^{98}$, O. Beltramello ${ }^{30}$, O. Benary ${ }^{153}$, D. Benchekroun ${ }^{135 a}$, M. Bender ${ }^{100}$, K. Bendtz ${ }^{146 a, 146 b}$, N. Benekos ${ }^{10}$, Y. Benhammou ${ }^{153}$, E. Benhar Noccioli49, J. A. Benitez Garcia ${ }^{159 b}$, D. P. Benjamin ${ }^{45}$, J. R. Bensinger ${ }^{23}$, S. Bentvelsen ${ }^{107}$, L. Beresford ${ }^{120}$, M. Beretta ${ }^{47}$, D. Berge ${ }^{107}$, E. Bergeaas Kuutmann ${ }^{166}$, N. Berger ${ }^{5}$, F. Berghaus ${ }^{169}$, J. Beringer ${ }^{15}$, C. Bernard ${ }^{22}$, N. R. Bernard ${ }^{86}$, C. Bernius ${ }^{110}$, F. U. Bernlochner ${ }^{21}$, T. Berry ${ }^{77}$, P. Berta ${ }^{129}$, C. Bertella ${ }^{83}$, G. Bertoli 146a,146b, F. Bertolucci ${ }^{124 a, 124 b}$, C. Bertsche ${ }^{113}$, D. Bertsche ${ }^{113}$, M. I. Besana ${ }^{91 a}$, G. J. Besjes ${ }^{36}$, O. Bessidskaia Bylund ${ }^{146 a, 146 b}$, M. Bessner ${ }^{42}$, N. Besson ${ }^{136}$, C. Betancourt ${ }^{48}$, S. Bethke ${ }^{101}$, A. J. Bevan ${ }^{76}$, W. Bhimji ${ }^{15}$, R. M. Bianchi ${ }^{125}$, L. Bianchini ${ }^{23}$, M. Bianco ${ }^{30}$, O. Biebel ${ }^{100}$, D. Biedermann ${ }^{16}$, S. P. Bieniek ${ }^{78}$, N. V. Biesuz ${ }^{124 a, 124 b}$, M. Biglietti ${ }^{134 a}$, J. Bilbao De Mendizabal ${ }^{49}$, H. Bilokon ${ }^{47}$, M. Bindi ${ }^{54}$, S. Binet ${ }^{117}$, A. Bingul ${ }^{19 b}$, C. Bini ${ }^{132 a, 132 b}$, S. Biondi20a,20b, D. M. Bjergaard ${ }^{45}$ ， C. W. Black ${ }^{150}$, J. E. Black ${ }^{143}$, K. M. Black ${ }^{22}$, D. Blackburn ${ }^{138}$, R. E. Blair ${ }^{6}$, J.-B. Blanchard ${ }^{136}$, J. E. Blanco ${ }^{77}$, T. Blazek ${ }^{144 a}, \quad$ I. Bloch ${ }^{42}$, C. Blocker ${ }^{23}$, W. Blum ${ }^{83, *}$ U. Blumenschein ${ }^{54}$, S. Blunier ${ }^{32 a}$, G. J. Bobbink ${ }^{107}$, V. S. Bobrovnikov ${ }^{109, \mathrm{c}}$, S. S. Bocchetta ${ }^{81}$, A. Bocci ${ }^{45}$, C. Bock ${ }^{100}$, M. Boehler ${ }^{48}$,

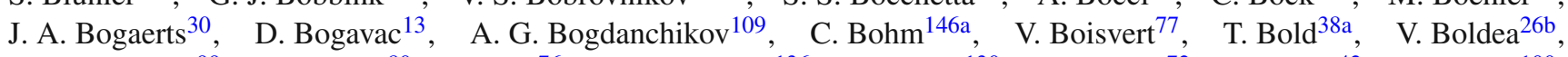
A. S. Boldyrev ${ }^{99}$, M. Bomben ${ }^{80}$, M. Bona ${ }^{76}$, M. Boonekamp ${ }^{136}$, A. Borisov ${ }^{130}$, G. Borissov ${ }^{72}$, S. Borroni $^{42}$, J. Bortfeldt ${ }^{100}$,

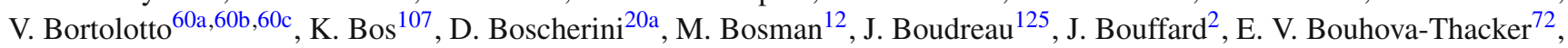
D. Boumediene ${ }^{34}$, C. Bourdarios ${ }^{117}$, N. Bousson ${ }^{114}$, S. K. Boutle ${ }^{53}$, A. Boveia ${ }^{30}$, J. Boyd ${ }^{30}$, I. R. Boyko ${ }^{65}$, I. Bozic ${ }^{13}$, J. Bracinik ${ }^{18}$, A. Brandt ${ }^{8}$, G. Brandt ${ }^{54}$, O. Brandt ${ }^{58 a}$, U. Bratzler ${ }^{156}$, B. Brau ${ }^{86}$, J. E. Brau ${ }^{116}$, H. M. Braun ${ }^{175, *}$, W. D. Breaden Madden ${ }^{53}$, K. Brendlinger ${ }^{122}$, A. J. Brennan ${ }^{88}$, L. Brenner ${ }^{107}$, R. Brenner ${ }^{166}$, S. Bressler ${ }^{172}$, T. M. Bristow ${ }^{46}$, D. Britton ${ }^{53}$, D. Britzger ${ }^{42}$, F. M. Brochu ${ }^{28}$, I. Brock ${ }^{21}$, R. Brock ${ }^{90}$, J. Bronner ${ }^{101}$, G. Brooijmans ${ }^{35}$, T. Brooks ${ }^{77}$, W. K. Brooks ${ }^{32 b}$, J. Brosamer ${ }^{15}$, E. Brost ${ }^{116}$, P. A. Bruckman de Renstrom ${ }^{39}$, D. Bruncko ${ }^{144 b}$, R. Bruneliere ${ }^{48}$, A. Bruni ${ }^{20 a}$, G. Bruni ${ }^{20 a}$, M. Bruschi ${ }^{20 a}$, N. Bruscino ${ }^{21}$, L. Bryngemark ${ }^{81}$, T. Buanes ${ }^{14}$, Q. Buat ${ }^{142}$, P. Buchholz ${ }^{141}$, A. G. Buckley ${ }^{53}$, S. I. Buda ${ }^{26 b}$, I. A. Budagov ${ }^{65}$, F. Buehrer ${ }^{48}$, L. Bugge ${ }^{119}$, M. K. Bugge ${ }^{119}$, O. Bulekov ${ }^{98}$, D. Bullock ${ }^{8}$, H. Burckhart ${ }^{30}$, S. Burdin ${ }^{74}$, C. D. Burgard ${ }^{48}$, B. Burghgrave ${ }^{108}$, S. Burke ${ }^{131}$, I. Burmeister ${ }^{43}$, E. Busato ${ }^{34}$, D. Büscher ${ }^{48}$, V. Büscher ${ }^{83}$, P. Bussey ${ }^{53}$, J. M. Butler ${ }^{22}$, A. I. Butt ${ }^{3}$, C. M. Buttar ${ }^{53}$, J. M. Butterworth ${ }^{78}$, P. Butti ${ }^{107}$, W. Buttinger ${ }^{25}$, A. Buzatu ${ }^{53}$, A. R. Buzykaev ${ }^{109, c}$, S. Cabrera Urbán ${ }^{167}$, D. Caforio ${ }^{128}$, V. M. Cairo ${ }^{37 a, 37 b}$, O. Cakir ${ }^{4 a}$, N. Calace ${ }^{49}$, P. Calafiura ${ }^{15}$, 
A. Calandri ${ }^{136}$, G. Calderini ${ }^{80}$, P. Calfayan ${ }^{100}$, L. P. Caloba ${ }^{24 a}$, D. Calvet ${ }^{34}$, S. Calvet ${ }^{34}$, R. Camacho Toro ${ }^{31}$, S. Camarda ${ }^{42}$, P. Camarri ${ }^{133 a}$,133b , D. Cameron ${ }^{119}$, R. Caminal Armadans ${ }^{165}$, S. Campana ${ }^{30}$, M. Campanelli ${ }^{78}$, A. Campoverde ${ }^{148}$, V. Canale ${ }^{104 a, 104 b}$, A. Canepa ${ }^{159 a}$, M. Cano Bret ${ }^{33 e}$, J. Cantero ${ }^{82}$, R. Cantrill ${ }^{126 a}$, T. Cao ${ }^{40}$, M. D. M. Capeans Garrido ${ }^{30}$,

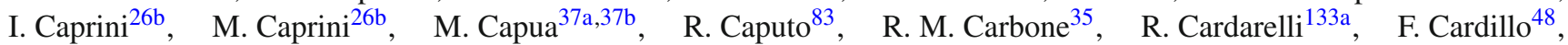
T. Carli $^{30}$ ， G. Carlino ${ }^{104 a}$ ， L. Carminati ${ }^{91 a}$,91b，S. Caron ${ }^{106}$ ，E. Carquin ${ }^{32 a}$ ， G. D. Carrillo-Montoya ${ }^{30}$ ，J. R. Carter ${ }^{28}$,

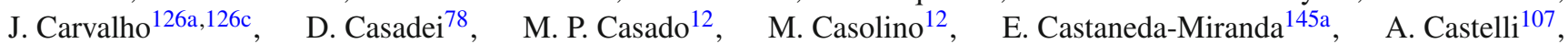
V. Castillo Gimenez ${ }^{167}$, N. F. Castro ${ }^{126 a, g}$, P. Catastini ${ }^{57}$, A. Catinaccio ${ }^{30}$, J. R. Catmore ${ }^{119}$, A. Cattai ${ }^{30}$, J. Caudron ${ }^{83}$, V. Cavaliere ${ }^{165}$, D. Cavalli ${ }^{91 a}$, M. Cavalli-Sforza ${ }^{12}$, V. Cavasinni ${ }^{124 a, 124 b}$, F. Ceradini ${ }^{134 a, 134 b}$, B. C. Cerio ${ }^{45}$, K. Cerny ${ }^{129}$, A. S. Cerqueira ${ }^{24 b}$, A. Cerri ${ }^{149}$, L. Cerrito ${ }^{76}$, F. Cerutti ${ }^{15}$, M. Cerv ${ }^{30}$, A. Cervelli17, S. A. Cetin ${ }^{19 c}$, A. Chafaq ${ }^{135 a}$, D. Chakraborty ${ }^{108}$, I. Chalupkova ${ }^{129}$, Y. L. Chan ${ }^{60 a}$, P. Chang ${ }^{165}$, J. D. Chapman ${ }^{28}$, D. G. Charlton ${ }^{18}$, C. C. Chau ${ }^{158}$, C. A. Chavez Barajas ${ }^{149}$, S. Cheatham ${ }^{152}$, A. Chegwidden ${ }^{90}$, S. Chekanov ${ }^{6}$, S. V. Chekulaev ${ }^{159 a}$, G. A. Chelkov ${ }^{65, h}$, M. A. Chelstowska ${ }^{89}$, C. Chen ${ }^{64}$, H. Chen ${ }^{25}$, K. Chen ${ }^{148}$, L. Chen ${ }^{33 d, i}$, S. Chen ${ }^{33 c}$, S. Chen ${ }^{155}$, X. Chen ${ }^{33 f}$, Y. Chen ${ }^{67}$, H. C. Cheng ${ }^{89}$, Y. Cheng ${ }^{31}$, A. Cheplakov ${ }^{65}$, E. Cheremushkina ${ }^{130}$, R. Cherkaoui El Moursli ${ }^{135 e}$, V. Chernyatin ${ }^{25, *}$, E. Cheu ${ }^{7}$, L. Chevalier ${ }^{136}$, V. Chiarella ${ }^{47}$, G. Chiarelli1 ${ }^{124 a, 124 b}$, G. Chiodini ${ }^{73 a}$, A. S. Chisholm ${ }^{18}$, R. T. Chislett ${ }^{78}$, A. Chitan ${ }^{26 b}$, M. V. Chizhov ${ }^{65}$, K. Choi $^{61}$, S. Chouridou ${ }^{9}$, B. K. B. Chow ${ }^{100}$, V. Christodoulou ${ }^{78}$, D. Chromek-Burckhart ${ }^{30}$, J. Chudoba ${ }^{127}$, A. J. Chuinard ${ }^{87}$, J. J. Chwastowski ${ }^{39}$, L. Chytka ${ }^{115}$, G. Ciapetti ${ }^{132 a, 132 b}$, A. K. Ciftci ${ }^{4 a}$, D. Cinca ${ }^{53}$, V. Cindro ${ }^{75}$, I. A. Cioara ${ }^{21}$, A. Ciocio ${ }^{15}$, F. Cirotto ${ }^{104 a, 104 b}$, Z. H. Citron ${ }^{172}$, M. Ciubancan ${ }^{26 b}$, A. Clark ${ }^{49}$, B. L. Clark ${ }^{57}$, P. J. Clark ${ }^{46}$, R. N. Clarke ${ }^{15}$, C. Clement ${ }^{146 a, 146 b}$, Y. Coadou ${ }^{85}$, M. Cobal ${ }^{164 a, 164 c}$, A. Coccaro ${ }^{49}$, J. Cochran ${ }^{64}$, L. Coffey ${ }^{23}$, J. G. Cogan ${ }^{143}$, L. Colasurdo ${ }^{106}$, B. Cole ${ }^{35}$, S. Cole ${ }^{108}$, A. P. Colijn ${ }^{107}$, J. Collot ${ }^{55}$, T. Colombo ${ }^{58 c}$, G. Compostella ${ }^{101}$, P. Conde Muiño ${ }^{126 a, 126 b}$, E. Coniavitis ${ }^{48}$, S. H. Connell ${ }^{145 b}$, I. A. Connelly ${ }^{77}$, V. Consorti ${ }^{48}$, S. Constantinescu ${ }^{26 b}$, C. Conta ${ }^{121 a, 121 b}$, G. Conti ${ }^{30}$, F. Conventi ${ }^{104 a, j}$, M. Cooke ${ }^{15}$, B. D. Cooper $^{78}$, A. M. Cooper-Sarkar ${ }^{120}$, T. Cornelissen ${ }^{175}$, M. Corradi ${ }^{20 a}$, F. Corriveau ${ }^{87, \mathrm{k}}$, A. Corso-Radu ${ }^{163}$, A. Cortes-Gonzalez ${ }^{12}$, G. Cortiana ${ }^{101}$, G. Costa ${ }^{91 a}$, M. J. Costa ${ }^{167}$, D. Costanzo ${ }^{139}$, D. Côté ${ }^{8}$, G. Cottin ${ }^{28}$, G. Cowan ${ }^{77}$, B. E. Cox $^{84}$, K. Cranmer ${ }^{110}$, G. Cree ${ }^{29}$, S. Crépé-Renaudin ${ }^{55}$, F. Crescioli ${ }^{80}$, W. A. Cribbs ${ }^{146 a, 146 b}$, M. Crispin Ortuzar ${ }^{120}, \quad$ M. Cristinziani ${ }^{21}, \quad$ V. Croft $^{106}, \quad$ G. Crosetti ${ }^{37 a, 37 b}$, T. Cuhadar Donszelmann ${ }^{139}$, J. Cummings ${ }^{176}$, M. Curatolo ${ }^{47}$, J. Cúth ${ }^{83}$, C. Cuthbert ${ }^{150}$, H. Czirr ${ }^{141}$, P. Czodrowski ${ }^{3}$, S. D’Auria ${ }^{53}$, M. D’Onofrio ${ }^{74}$, M. J. Da Cunha Sargedas De Sousa ${ }^{126 a, 126 b}$, C. Da Via ${ }^{84}$, W. Dabrowski ${ }^{38 a}$, A. Dafinca ${ }^{120}$, T. Dai ${ }^{89}$, O. Dale ${ }^{14}$, F. Dallaire ${ }^{95}$, C. Dallapiccola ${ }^{86}$, M. Dam ${ }^{36}$, J. R. Dandoy ${ }^{31}$, N.P. Dang ${ }^{48}$, A. C. Daniells ${ }^{18}$, M. Danninger ${ }^{168}$, M. Dano Hoffmann ${ }^{136}$, V. Dao ${ }^{48}$, G. Darbo ${ }^{50 a}$, S. Darmora ${ }^{8}$, J. Dassoulas ${ }^{3}$, A. Dattagupta ${ }^{61}$, W. Davey ${ }^{21}$ ， C. David ${ }^{169}$ ， T. Davidek ${ }^{129}$, E. Davies ${ }^{120,1}$, M. Davies ${ }^{153}$, P. Davison ${ }^{78}$, Y. Davygora ${ }^{58 a}$, E. Dawe ${ }^{88}$, I. Dawson ${ }^{139}$, R. K. Daya-Ishmukhametova ${ }^{86}$, K. De ${ }^{8}$, R. de Asmundis ${ }^{104 a}$, A. De Benedetti ${ }^{113}$, S. De Castro ${ }^{20 a, 20 b}$, S. De $\mathrm{Cecco}^{80}$, N. De Groot ${ }^{106}$, P. de Jong ${ }^{107}$, H. De la Torre ${ }^{82}$, F. De Lorenzi ${ }^{64}$, D. De Pedis ${ }^{132 a}$, A. De Salvo ${ }^{132 a}$, U. De Sanctis ${ }^{149}$, A. De Santo ${ }^{149}$ ， J. B. De Vivie De Regie ${ }^{117}$, W. J. Dearnaley ${ }^{72}$, R. Debbe ${ }^{25}$, C. Debenedetti ${ }^{137}$, D. V. Dedovich ${ }^{65}$, I. Deigaard ${ }^{107}$, J. Del Peso ${ }^{82}$, T. Del Prete ${ }^{124 a, 124 b}$, D. Delgove ${ }^{117}$, F. Deliot ${ }^{136}$, C. M. Delitzsch ${ }^{49}$, M. Deliyergiyev ${ }^{75}$, A. Dell'Acqua ${ }^{30}$, L. Dell'Asta ${ }^{22}$, M. Dell'Orso ${ }^{124 a, 124 b}$, M. Della Pietra ${ }^{104 a, j}$, D. della Volpe ${ }^{49}$, M. Delmastro5, P. A. Delsart55, C. Deluca ${ }^{57}$, D. A. DeMarco ${ }^{158}$, S. Demers ${ }^{176}$, M. Demichev ${ }^{65}$, A. Demilly ${ }^{80}$, S. P. Denisov ${ }^{130}$, D. Derendarz ${ }^{39}$, J. E. Derkaoui ${ }^{135 d}$, F. Derue ${ }^{80}$, P. Dervan ${ }^{74}$, K. Desch $^{21}$, C. Deterre ${ }^{42}$, K. Dette ${ }^{43}$, P. O. Deviveiros ${ }^{30}$, A. Dewhurst ${ }^{131}$, S. Dhaliwal ${ }^{23}$, A. Di Ciaccio ${ }^{133 a, 133 b}$, L. Di Ciaccio ${ }^{5}$, A. Di Domenico ${ }^{132 a, 132 b}$, C. Di Donato 104a,104b, A. Di Girolamo ${ }^{30}$, B. Di Girolamo ${ }^{30}$, A. Di Mattia ${ }^{152}$, B. Di Micco 134a,134b, R. Di Nardo ${ }^{47}$, A. Di Simone ${ }^{48}$, R. Di Sipio ${ }^{158}$, D. Di Valentino ${ }^{29}, \quad$ C. Diaconu ${ }^{85}, \quad$ M. Diamond ${ }^{158}$, F. A. Dias ${ }^{46}, \quad$ M. A. Diaz ${ }^{32 a}$, E. B. Diehl ${ }^{89}$, J. Dietrich ${ }^{16}$, S. Diglio ${ }^{85}$, A. Dimitrievska ${ }^{13}$, J. Dingfelder ${ }^{21}$, P. Dita ${ }^{26 b}$, S. Dita $^{26 b}$, F. Dittus ${ }^{30}$, F. Djama ${ }^{85}$,

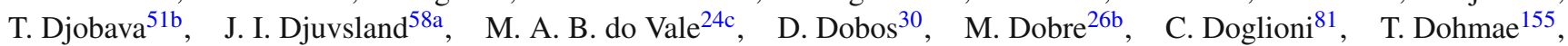
J. Dolejsi ${ }^{129}$, Z. Dolezal ${ }^{129}$, B. A. Dolgoshein ${ }^{98, *}$, M. Donadelli24d, S. Donati124a,124b, P. Dondero ${ }^{121 a, 121 b}$, J. Donini ${ }^{34}$, J. Dopke ${ }^{131}$, A. Doria ${ }^{104 a}$, M. T. Dova ${ }^{71}$, A. T. Doyle ${ }^{53}$, E. Drechsler ${ }^{54}$, M. Dris ${ }^{10}$, E. Dubreuil ${ }^{34}$, E. Duchovni ${ }^{172}$, G. Duckeck ${ }^{100}$, O. A. Ducu ${ }^{26 b, 85}$, D. Duda ${ }^{107}$, A. Dudarev ${ }^{30}$, L. Duflot ${ }^{117}$, L. Duguid ${ }^{77}$, M. Dührssen ${ }^{30}$, M. Dunford ${ }^{58 a}$, H. Duran Yildiz ${ }^{4 a}$, M. Düren ${ }^{52}, \quad$ A. Durglishvili ${ }^{51 b}, \quad$ D. Duschinger ${ }^{44}, \quad$ B. Dutta ${ }^{42}, \quad$ M. Dyndal ${ }^{38 a}, \quad$ C. Eckardt ${ }^{42}$, K. M. Ecker ${ }^{101}$, R. C. Edgar ${ }^{89}$, W. Edson ${ }^{2}$, N. C. Edwards ${ }^{46}$, W. Ehrenfeld ${ }^{21}$, T. Eifert ${ }^{30}$, G. Eigen ${ }^{14}$, K. Einsweiler ${ }^{15}$, T. Ekelof ${ }^{166}$, M. El Kacimi ${ }^{135 c}$, M. Ellert ${ }^{166}$, S. Elles $^{5}$, F. Ellinghaus ${ }^{175}$, A. A. Elliot ${ }^{169}$, N. Ellis ${ }^{30}$, J. Elmsheuser ${ }^{100}$, M. Elsing ${ }^{30}$, D. Emeliyanov ${ }^{131}$, Y. Enari ${ }^{155}$, O. C. Endner ${ }^{83}$, M. Endo ${ }^{118}$, J. Erdmann ${ }^{43}$, A. Ereditato ${ }^{17}$, G. Ernis ${ }^{175}$, J. Ernst ${ }^{2}$, M. Ernst ${ }^{25}$, S. Errede ${ }^{165}$, E. Ertel ${ }^{83}$, M. Escalier ${ }^{117}$, H. Esch ${ }^{43}$, C. Escobar ${ }^{125}$, B. Esposito ${ }^{47}$, A. I. Etienvre ${ }^{136}$,

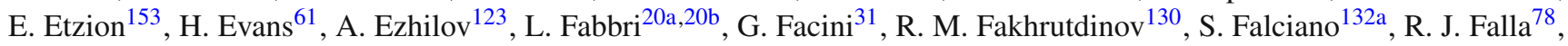
J. Faltova ${ }^{129}$, Y. Fang ${ }^{33 a}$, M. Fanti ${ }^{91 a}$,91b , A. Farbin ${ }^{8}$, A. Farilla ${ }^{134 a}$, T. Farooque ${ }^{12}$, S. Farrell ${ }^{15}$, S. M. Farrington ${ }^{170}$, P. Farthouat ${ }^{30}$, F. Fassi ${ }^{135 e}$, P. Fassnacht ${ }^{30}$, D. Fassouliotis ${ }^{9}$, M. Faucci Giannelli7, A. Favareto ${ }^{50 a, 50 b}$, L. Fayard ${ }^{117}$, O. L. Fedin ${ }^{123, \mathrm{~m}}$, W. Fedorko ${ }^{168}$, S. Feigl ${ }^{30}$, L. Feligioni ${ }^{85}$, C. Feng ${ }^{33 d}$, E. J. Feng ${ }^{30}$, H. Feng ${ }^{89}$, A. B. Fenyuk ${ }^{130}$, 
L. Feremenga ${ }^{8}$, P. Fernandez Martinez ${ }^{167}$, S. Fernandez Perez ${ }^{30}$, J. Ferrando ${ }^{53}$, A. Ferrari ${ }^{166}$, P. Ferrari ${ }^{107}$, R. Ferrari ${ }^{121 a}$, D. E. Ferreira de Lima $^{53}$, A. Ferrer ${ }^{167}$, D. Ferrere ${ }^{49}$, C. Ferretti ${ }^{89}$, A. Ferretto Parodi ${ }^{50,50 b}$, M. Fiascaris ${ }^{31}$, F. Fiedler ${ }^{83}$,

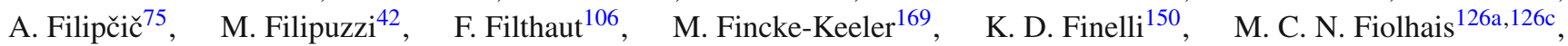
L. Fiorini ${ }^{167}$, A. Firan ${ }^{40}$, A. Fischer ${ }^{2}$, C. Fischer ${ }^{12}$, J. Fischer ${ }^{175}$, W. C. Fisher ${ }^{90,}$ N. Flaschel ${ }^{42}$, I. Fleck ${ }^{141}$, P. Fleischmann ${ }^{89}$, G. T. Fletcher ${ }^{139}$, G. Fletcher ${ }^{76}$, R. R. M. Fletcher ${ }^{122}$, T. Flick ${ }^{175}$, A. Floderus ${ }^{81}$, L. R. Flores Castillo ${ }^{60 a}$, M. J. Flowerdew ${ }^{101}$, A. Formica ${ }^{136}$, A. Forti ${ }^{84}$, D. Fournier ${ }^{117}$, H. Fox ${ }^{72}$, S. Fracchia ${ }^{12}$, P. Francavilla ${ }^{80}$, M. Franchini ${ }^{20 a, 20 b}$,

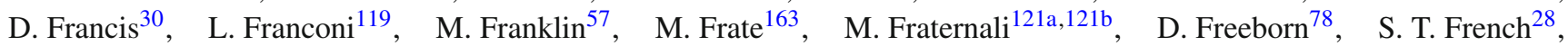
F. Friedrich ${ }^{44}$, D. Froidevaux ${ }^{30}$, J. A. Frost ${ }^{120}$, C. Fukunaga ${ }^{156}$, E. Fullana Torregrosa ${ }^{83}$, B. G. Fulsom ${ }^{143}$, T. Fusayasu ${ }^{102}$, J. Fuster ${ }^{167}$ ， C. Gabaldon ${ }^{55}$ O. Gabizon ${ }^{175}$, A. Gabrielli ${ }^{20 a, 20 b}, \quad$ A. Gabrielli ${ }^{15}$ ， G. P. Gach ${ }^{18}, \quad$ S. Gadatsch ${ }^{30}$, S. Gadomski ${ }^{49}$, G. Gagliardi ${ }^{50,50 b}$, P. Gagnon ${ }^{61}$, C. Galea ${ }^{106}$, B. Galhardo ${ }^{126 a, 126 c}$, E. J. Gallas ${ }^{120}$, B. J. Gallop ${ }^{131}$, P. Gallus ${ }^{128}$, G. Galster ${ }^{36}$, K. K. Gan ${ }^{111}$, J. Gao ${ }^{33 b, 85}$, Y. Gao ${ }^{46}$, Y. S. Gao ${ }^{143, e}$, F. M. Garay Walls ${ }^{46}$, F. Garberson ${ }^{176}$, C. García ${ }^{167}$, J. E. García Navarro ${ }^{167}$, M. Garcia-Sciveres ${ }^{15}$, R. W. Gardner ${ }^{31}$, N. Garelli ${ }^{143}$, V. Garonne ${ }^{119}$, C. Gattii ${ }^{47}$, A. Gaudiello ${ }^{50 a, 50 b}$, G. Gaudio ${ }^{121 a}$, B. Gaur ${ }^{141}$, L. Gauthier ${ }^{95}$, P. Gauzzi132a,132b, I. L. Gavrilenko ${ }^{96}$, C. Gay ${ }^{168}$, G. Gaycken ${ }^{21}$, E. N. Gazis ${ }^{10}$, P. Ge ${ }^{33 d}$, Z. Gecse ${ }^{168}$, C. N. P. Gee ${ }^{131}$, Ch. Geich-Gimbel ${ }^{21}$, M. P. Geisler ${ }^{58 a}$, C. Gemme ${ }^{50 a}$,

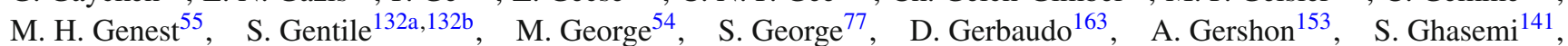
H. Ghazlane ${ }^{135 b}$, B. Giacobbe ${ }^{20 a}$, S. Giagu ${ }^{132 a, 132 b}$, V. Giangiobbe ${ }^{12}$, P. Giannetti ${ }^{124 a, 124 b}$, B. Gibbard ${ }^{25}$, S. M. Gibson ${ }^{77}$, M. Gignac ${ }^{168}$, M. Gilchriese ${ }^{15}$, T. P. S. Gillam ${ }^{28}, \quad$ D. Gillberg ${ }^{30}, \quad$ G. Gilles ${ }^{34}, \quad$ D. M. Gingrich ${ }^{3, d}$, N. Giokaris ${ }^{9}$, M. P. Giordani ${ }^{164 a, 164 c}$, F. M. Giorgi ${ }^{20 a}$, F. M. Giorgi ${ }^{16}$, P. F. Giraud ${ }^{136}$, P. Girominii7 ${ }^{47}$ D. Giugni ${ }^{91 a}$, C. Giuliani ${ }^{101}$, M. Giulini ${ }^{58 b}$, B. K. Gjelsten ${ }^{119}$, S. Gkaitatzis ${ }^{154}$, I. Gkialas ${ }^{154}$, E. L. Gkougkousis ${ }^{117}$, L. K. Gladilinn ${ }^{99}$, C. Glasman ${ }^{82}$, J. Glatzer ${ }^{30}$, P. C. F. Glaysher ${ }^{46}$, A. Glazov ${ }^{42}$, M. Goblirsch-Kolb ${ }^{101}$, J. R. Goddard ${ }^{76}$, J. Godlewskii ${ }^{39}$, S. Goldfarb ${ }^{89}$, T. Golling ${ }^{49}$, D. Golubkov ${ }^{130}$, A. Gomes ${ }^{126 a, 126 b, 126 d, ~ R . ~ G o n c ̧ a l o ~}{ }^{126 a}$, J. Goncalves Pinto Firmino Da Costa ${ }^{136}$, L. Gonella ${ }^{21}$, S. González de la Hoz ${ }^{167}$, G. Gonzalez Parra ${ }^{12}$, S. Gonzalez-Sevilla ${ }^{49}$, L. Goossens ${ }^{30}$, P. A. Gorbounov ${ }^{97}$, H. A. Gordon ${ }^{25}$, I. Gorelov ${ }^{105}$, B. Gorini ${ }^{30}$, E. Gorini ${ }^{73 a, 73 b}$, A. Gorišek ${ }^{75}$, E. Gornicki ${ }^{39}$, A. T. Goshaw ${ }^{45}$, C. Gössling ${ }^{43}$, M. I. Gostkin ${ }^{65}$, D. Goujdami ${ }^{135 c}$, A. G. Goussiou ${ }^{138}$, N. Govender ${ }^{145 b}$, E. Gozani ${ }^{152}$, H. M. X. Grabas ${ }^{137}$, L. Graber ${ }^{54}$, I. Grabowska-Bold ${ }^{38 a}$, P. O. J. Gradin ${ }^{166}, \quad$ P. Grafström ${ }^{20 a, 20 b}, \quad$ J. Gramling ${ }^{49}$, E. Gramstad ${ }^{119}$, S. Grancagnolo ${ }^{16}$, V. Gratchev ${ }^{123}$, H. M. Gray ${ }^{30}$, E. Graziani ${ }^{134 a}$, Z. D. Greenwood ${ }^{79, n}$, C. Grefe ${ }^{21}$, K. Gregersen ${ }^{78}$, I. M. Gregor ${ }^{42}$, P. Grenier ${ }^{143}$, J. Griffiths ${ }^{8}$, A. A. Grillo ${ }^{137}$, K. Grimm ${ }^{72}$, S. Grinstein ${ }^{12, o}$, Ph. Gris ${ }^{34}$, J.-F. Grivaz ${ }^{117}$, J. P. Grohs ${ }^{44}$, A. Grohsjean ${ }^{42}$, E. Gross ${ }^{172}$, J. Grosse-Knetter ${ }^{54}$, G. C. Grossi ${ }^{79}$, Z. J. Grout ${ }^{149}$, L. Guan ${ }^{89}$, J. Guenther ${ }^{128}$, F. Guescini4 ${ }^{49}$, D. Guest ${ }^{163}$, O. Gueta ${ }^{153}$, E. Guido ${ }^{50 a, 50 b}$, T. Guillemin ${ }^{117}$, S. Guindon ${ }^{2}$, U. Gul ${ }^{53}$, C. Gumpert ${ }^{44}$, J. Guo ${ }^{33 e}$, Y. Guo ${ }^{33 b, p}$, S. Gupta ${ }^{120}$, G. Gustavino ${ }^{132 a, 132 b}$, P. Gutierrez ${ }^{113}$, N. G. Gutierrez Ortiz ${ }^{78}$, C. Gutschow ${ }^{44}$,

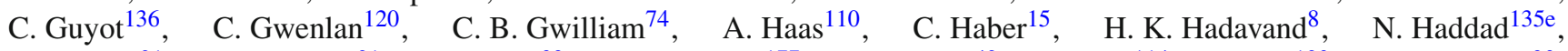
P. Haefner ${ }^{21}$ ， S. Hageböck ${ }^{21}$ ， Z. Hajduk ${ }^{39}$, H. Hakobyan ${ }^{177}$, M. Haleem ${ }^{42}$, J. Haley ${ }^{114}$, D. Hall ${ }^{120}$, G. Halladjian ${ }^{90}$, G. D. Hallewell ${ }^{85}$, K. Hamacher ${ }^{175}$, P. Hamal ${ }^{115}$, K. Hamano ${ }^{169}$, A. Hamilton ${ }^{145 a}$, G. N. Hamity ${ }^{139}$, P. G. Hamnett ${ }^{42}$, L. Han ${ }^{33 b}$ ， K. Hanagaki ${ }^{66, q}$ ， K. Hanawa ${ }^{155}$ ， M. Hance ${ }^{137}$ ， B. Haney ${ }^{122}$, P. Hanke ${ }^{58 a}$ ， R. Hanna ${ }^{136}$, J. B. Hansen ${ }^{36}$,

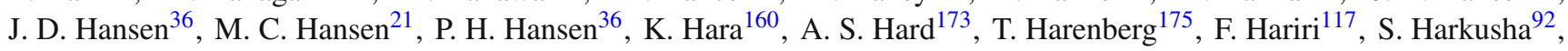

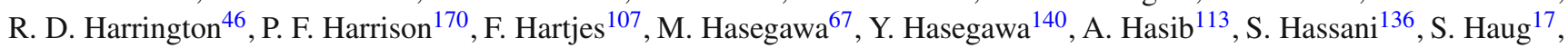
R. Hauser ${ }^{90}$, L. Hauswald ${ }^{44}$, M. Havranek ${ }^{127}$, C. M. Hawkes ${ }^{18}$, R. J. Hawkings ${ }^{30}$, A. D. Hawkins ${ }^{81}$, T. Hayashi ${ }^{160}$,

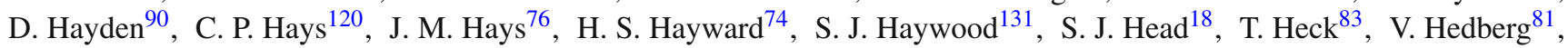
L. Heelan ${ }^{8}$, S. Heim ${ }^{122}$, T. Heim ${ }^{175}$, B. Heinemann ${ }^{15}$, L. Heinrich ${ }^{110}$, J. Hejbal ${ }^{127}$, L. Helary ${ }^{22}$, S. Hellman ${ }^{146 a, 146 b}$, D. Hellmich $^{21}$, C. Helsens ${ }^{12}$, J. Henderson ${ }^{120}$, R. C. W. Henderson ${ }^{72}$, Y. Heng ${ }^{173}$, C. Hengler ${ }^{42}$, S. Henkelmann ${ }^{168}$, A. Henrichs ${ }^{176}$, A. M. Henriques Correia ${ }^{30}$, S. Henrot-Versille ${ }^{117}$, G. H. Herbert ${ }^{16}$, Y. Hernández Jiménez ${ }^{167}$, G. Herten ${ }^{48}$, R. Hertenberger ${ }^{100}$, L. Hervas ${ }^{30}$, G. G. Hesketh ${ }^{78}$, N. P. Hessey ${ }^{107}$, J. W. Hetherly ${ }^{40}$, R. Hickling ${ }^{76}$, E. Higón-Rodriguez ${ }^{167}$, E. Hill ${ }^{169}$ ， J. C. Hill ${ }^{28}$ ， K. H. Hiller ${ }^{42}$, S. J. Hillier ${ }^{18}$, I. Hinchliffe ${ }^{15}$, E. Hines ${ }^{122}$, R. R. Hinman ${ }^{15}$, M. Hirose ${ }^{157}$, D. Hirschbuehl ${ }^{175}$, J. Hobbs ${ }^{148}$, N. Hod ${ }^{107}$, M. C. Hodgkinson ${ }^{139}$, P. Hodgson ${ }^{139}$, A. Hoecker ${ }^{30}$, M. R. Hoeferkamp ${ }^{105}$, F. Hoenig ${ }^{100}$, M. Hohlfeld ${ }^{83}$, D. Hohn ${ }^{21}$, T. R. Holmes ${ }^{15}$, M. Homann ${ }^{43}$, T. M. Hong ${ }^{125}$, W. H. Hopkins ${ }^{116}$, Y. Horii ${ }^{103}$, A. J. Horton ${ }^{142}$ ， J-Y. Hostachy ${ }^{55}$ ， S. Hou ${ }^{151}$ ， A. Hoummada ${ }^{135 a}$ ， J. Howard ${ }^{120}$, J. Howarth ${ }^{42}, \quad$ M. Hrabovsky ${ }^{115}$, I. Hristova ${ }^{16}$, J. Hrivnac ${ }^{117}$, T. Hryn'ova ${ }^{5}$, A. Hrynevich ${ }^{93}$, C. Hsu ${ }^{145 c}$, P. J. Hsu ${ }^{151, r}$, S.-C. Hsu ${ }^{138}$, D. Hu ${ }^{35}$, Q. Hu ${ }^{33 b}$, X. Hu ${ }^{89}$, Y. Huang ${ }^{42}$, Z. Hubacek ${ }^{128}$, F. Hubaut ${ }^{85}$, F. Huegging ${ }^{21}$, T. B. Huffman ${ }^{120}$, E. W. Hughes ${ }^{35}$, G. Hughes ${ }^{72}$,

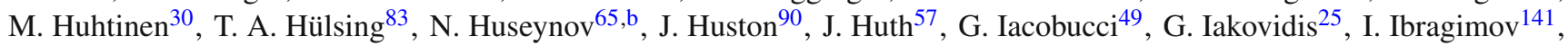
L. Iconomidou-Fayard ${ }^{117}$, E. Ideal ${ }^{176}$, Z. Idrissi ${ }^{135 e}$, P. Iengo $^{30}$, O. Igonkina ${ }^{107}$, T. Iizawa $^{171}$, Y. Ikegami ${ }^{66}$, K. Ikematsu $^{141}$, M. Ikeno ${ }^{66}$, Y. Ilchenko ${ }^{31, \mathrm{~s}}$, D. Iliadis ${ }^{154}$, N. Ilic ${ }^{143}$, T. Ince ${ }^{101}$ ， G. Introzzi ${ }^{121 a, 121 b}$, P. Ioannou9 , M. Iodice ${ }^{134 a}$, K. Iordanidou ${ }^{35}$, V. Ippolito ${ }^{57}$, A. Irles Quiles ${ }^{167}$, C. Isaksson ${ }^{166}$, M. Ishino ${ }^{68}$, M. Ishitsuka ${ }^{157}$, R. Ishmukhametov ${ }^{111}$, C. Issever ${ }^{120}$, S. Istin ${ }^{19 a}$, J. M. Iturbe Ponce ${ }^{84}$, R. Iuppa ${ }^{133 a, 133 b}$, J. Ivarsson ${ }^{81}$, W. Iwanski ${ }^{39}$, H. Iwasaki ${ }^{66}$, J. M. Izen ${ }^{41}$, 
V. Izzo ${ }^{104 a}$ ，S. Jabbar ${ }^{3}$ ， B. Jackson ${ }^{122}$ ， M. Jackson ${ }^{74}$ ，P. Jackson ${ }^{1}$ ， M. R. Jaekel ${ }^{30}$ ，V. Jain²，K. Jakobs ${ }^{48}$ ， S. Jakobsen ${ }^{30}$, T. Jakoubek ${ }^{127}$ ，J. Jakubek ${ }^{128}$ ， D. O. Jamin ${ }^{114}$ ， D. K. Jana ${ }^{79}$ ， E. Jansen ${ }^{78} ， \quad$ R. Jansky ${ }^{62}$ ， J. Janssen ${ }^{21}$ ， M. Janus ${ }^{54}$, G. Jarlskog ${ }^{81}$ ， N. Javadov ${ }^{65, b}$ ， T. Javůrek ${ }^{48}$ ， L. Jeanty ${ }^{15}$ ， J. Jejelava ${ }^{51 a, t}$ ， G.-Y. Jeng ${ }^{150 ， \quad D . ~ J e n n e n s ~}{ }^{88}$ ， P. Jenni ${ }^{48, u}$, J. Jentzsch ${ }^{43}$ ， C. Jeske ${ }^{170}$ ， S. Jézéquel ${ }^{5}$ ，H. Ji ${ }^{173}$ ， J. Jia ${ }^{148}$ ， Y. Jiang ${ }^{33 b} ，$ S. Jiggins ${ }^{78}$ ， J. Jimenez Pena ${ }^{167}$ ， S. Jin ${ }^{33 a}$, A. Jinaru ${ }^{26 b}$, O. Jinnouchi ${ }^{157}$, M. D. Joergensen ${ }^{36}$, P. Johansson ${ }^{139}$, K. A. Johns ${ }^{7}$, W. J. Johnson ${ }^{138}$, K. Jon-And ${ }^{146 a, 146 b}$, G. Jones ${ }^{170}$, R. W. L. Jones ${ }^{72}$, T. J. Jones ${ }^{74}$, J. Jongmanns ${ }^{58 a}$, P. M. Jorge ${ }^{126 a, 126 b}$, K. D. Joshi ${ }^{84}$, J. Jovicevic ${ }^{159 a}$, X. Ju ${ }^{173}$, P. Jussel $^{62}$, A. Juste Rozas ${ }^{12, o}$, M. Kaci ${ }^{167}$, A. Kaczmarska ${ }^{39}$, M. Kado ${ }^{117}$, H. Kagan ${ }^{111}$, M. Kagan ${ }^{143}$, S. J. Kahn ${ }^{85}$, E. Kajomovitz ${ }^{45}$, C. W. Kalderon ${ }^{120}$, S. Kama ${ }^{40}$, A. Kamenshchikov ${ }^{130}$, N. Kanaya ${ }^{155}$, S. Kaneti ${ }^{28}$, V. A. Kantserov ${ }^{98}$, J. Kanzaki ${ }^{66}$, B. Kaplan ${ }^{110}$, L. S. Kaplan ${ }^{173}$, A. Kapliy ${ }^{31}$, D. Kar ${ }^{145 c}$, K. Karakostas ${ }^{10}$, A. Karamaoun ${ }^{3}$, N. Karastathis ${ }^{10,107}$, M. J. Kareem ${ }^{54}$, E. Karentzos ${ }^{10}$, M. Karnevskiy ${ }^{83}$, S. N. Karpov ${ }^{65}$, Z. M. Karpova ${ }^{65}$, K. Karthik ${ }^{110}$, V. Kartvelishvilii2 ${ }^{72}$, A. N. Karyukhin ${ }^{130}$, K. Kasahara ${ }^{160}$, L. Kashif ${ }^{173}$, R. D. Kass ${ }^{111}$, A. Kastanas ${ }^{14}$, Y. Kataoka ${ }^{155}$, C. Kato ${ }^{155}$, A. Katre ${ }^{49}$, $\begin{array}{lll}\text { J. Katzy } & 42\end{array}$ K. Kawade ${ }^{103}$ ， K. Kawagoe ${ }^{70}$, T. Kawamoto ${ }^{155}$ ， G. Kawamura ${ }^{54}$, S. Kazama ${ }^{155}$, V. F. Kazanin ${ }^{109, c}$, R. Keeler ${ }^{169}$, R. Kehoe ${ }^{40}$, J. S. Keller ${ }^{42}$, J. J. Kempster ${ }^{77}$, H. Keoshkerian ${ }^{84}$, O. Kepka ${ }^{127}$, B. P. Kerševan ${ }^{75}$, S. Kersten ${ }^{175}$, R. A. Keyes ${ }^{87}$, F. Khalil-zada ${ }^{11}$, H. Khandanyan ${ }^{146 a, 146 b}$, A. Khanov ${ }^{114}$, A. G. Kharlamov ${ }^{109, c}$, T. J. Khoo ${ }^{28}$, V. Khovanskiy ${ }^{97}$, E. Khramov ${ }^{65}$, J. Khubua ${ }^{51 b, v}$, S. Kido ${ }^{67}$, H. Y. Kim ${ }^{8}$, S. H. Kim ${ }^{160}$, Y. K. Kim ${ }^{31}$, N. Kimura ${ }^{154}$, O. M. Kind ${ }^{16}$, B. T. King ${ }^{74}$, M. King ${ }^{167}$, S. B. King ${ }^{168}$, J. Kirk ${ }^{131}$, A. E. Kiryunin ${ }^{101}$, T. Kishimoto ${ }^{67}$, D. Kisielewska ${ }^{38 a}$, F. Kiss ${ }^{48}$, K. Kiuchi ${ }^{160}$, O. Kivernyk ${ }^{136}$, E. Kladiva ${ }^{144 b}$, M. H. Klein ${ }^{35}$, M. Klein ${ }^{74}$, U. Klein ${ }^{74}$, K. Kleinknecht ${ }^{83}$, P. Klimek ${ }^{146 a, 146 b}$, A. Klimentov ${ }^{25}$, R. Klingenberg ${ }^{43}$, J. A. Klinger ${ }^{139}$, T. Klioutchnikova ${ }^{30}$, E.-E. Kluge ${ }^{58 a}$, P. Kluit ${ }^{107}$,

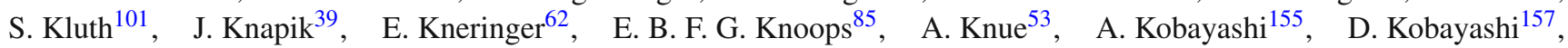
T. Kobayashi ${ }^{155}$, M. Kobel ${ }^{44}$, M. Kocian ${ }^{143}$, P. Kodys ${ }^{129}$, T. Koffas ${ }^{29}$, E. Koffeman ${ }^{107}$, L. A. Kogan ${ }^{120}$, S. Kohlmann ${ }^{175}$, Z. Kohout ${ }^{128}$, T. Kohriki ${ }^{66}$, T. Koi ${ }^{143}$, H. Kolanoski ${ }^{16}$, M. Kolb ${ }^{58 b}$, I. Koletsou ${ }^{5}$, A. A. Komar ${ }^{96, *}$, Y. Komori ${ }^{155}$, T. Kondo ${ }^{66}$, N. Kondrashova ${ }^{42}$, K. Köneke ${ }^{48}$, A. C. König ${ }^{106}$, T. Kono ${ }^{66}$, R. Konoplich ${ }^{110, w}$, N. Konstantinidis ${ }^{78}$, R. Kopeliansky ${ }^{152}$, S. Koperny ${ }^{38 a}$, L. Köpke ${ }^{83}$, A. K. Kopp ${ }^{48}$, K. Korcyl ${ }^{39}$, K. Kordas ${ }^{154}$, A. Korn ${ }^{78}$, A. A. Korol ${ }^{109, c}$, I. Korolkov ${ }^{12}$, E. V. Korolkova ${ }^{139}$, O. Kortner ${ }^{101}$, S. Kortner ${ }^{101}$, T. Kosek ${ }^{129}$, V. V. Kostyukhin ${ }^{21}$, V. M. Kotov ${ }^{65}$, A. Kotwal ${ }^{45}$, A. Kourkoumeli-Charalampidi ${ }^{154}$, C. Kourkoumelis ${ }^{9}$, V. Kouskoura ${ }^{25}$, A. Koutsman ${ }^{159 a}$, R. Kowalewski ${ }^{169}$, T. Z. Kowalski ${ }^{38 a}$, W. Kozanecki ${ }^{136}$, A. S. Kozhin ${ }^{130}$, V. A. Kramarenko ${ }^{99}$, G. Kramberger ${ }^{75}$, D. Krasnopevtsev ${ }^{98}$, M. W. Krasny ${ }^{80}$, A. Krasznahorkay ${ }^{30}$, J. K. Kraus ${ }^{21}$, A. Kravchenko ${ }^{25}$, S. Kreiss ${ }^{110}$, M. Kretz ${ }^{58 c}$, J. Kretzschmar ${ }^{74}$, K. Kreutzfeldt ${ }^{52}$, P. Krieger ${ }^{158}$, K. Krizka ${ }^{31}$, K. Kroeninger ${ }^{43}$, H. Kroha ${ }^{101}$, J. Kroll ${ }^{122}$, J. Kroseberg ${ }^{21}$, J. Krstic ${ }^{13}$, U. Kruchonak ${ }^{65}$, H. Krüger ${ }^{21}$, N. Krumnack ${ }^{64}$, A. Kruse ${ }^{173}$, M. C. Kruse ${ }^{45}$, M. Kruskal ${ }^{22}$, T. Kubota ${ }^{88}$, H. Kucuk ${ }^{78}$, S. Kuday ${ }^{4 b}$, S. Kuehn ${ }^{48}$, A. Kugel ${ }^{58 c}$, F. Kuger ${ }^{174}$, A. Kuhl ${ }^{137}$, T. Kuhl ${ }^{42}$, V. Kukhtin ${ }^{65}$, R. Kukla ${ }^{136}$, Y. Kulchitsky ${ }^{92}$, S. Kuleshov ${ }^{32 b}$, M. Kuna ${ }^{132 a, 132 b}$, T. Kunigo ${ }^{68}, \quad$ A. Kupco ${ }^{127}, \quad$ H. Kurashige ${ }^{67}$, Y. A. Kurochkin ${ }^{92}$, V. Kus ${ }^{127}$, E. S. Kuwertz ${ }^{169}$, M. Kuze ${ }^{157}$, J. Kvita ${ }^{115}$, T. Kwan ${ }^{169}$, D. Kyriazopoulos ${ }^{139}$, A. La Rosa ${ }^{137}$, J. L. La Rosa Navarro ${ }^{24 d}$, L. La Rotonda ${ }^{37 a, 37 b}$, C. Lacasta ${ }^{167}$, F. Lacava132a,132b, J. Lacey ${ }^{29}, \quad$ H. Lacker ${ }^{16}$, D. Lacour ${ }^{80}$, V. R. Lacuesta ${ }^{167}$, E. Ladygin ${ }^{65}$, R. Lafaye ${ }^{5}$, B. Laforge ${ }^{80}$, T. Lagouri ${ }^{176}$, S. Lai ${ }^{54}$, L. Lambourne ${ }^{78}$, S. Lammers ${ }^{61}$, C. L. Lampen ${ }^{7}$, W. Lampl ${ }^{7}$, E. Lançon ${ }^{136}$, U. Landgraf ${ }^{48}$, M. P. J. Landon ${ }^{76}$, V. S. Lang ${ }^{58 a}$, J. C. Lange ${ }^{12}$, A. J. Lankford ${ }^{163}$, F. Lanni ${ }^{25}$, K. Lantzsch ${ }^{21}$, A. Lanza ${ }^{121 a}$, S. Laplace ${ }^{80}$, C. Lapoire ${ }^{30}$, J. F. Laporte ${ }^{136}$, T. Lari ${ }^{91 a}$, F. Lasagni Manghi ${ }^{20 a, 20 b}$, M. Lassnig $^{30}$, P. Laurelli ${ }^{47}$, W. Lavrijsen ${ }^{15}$, A. T. Law ${ }^{137}$, P. Laycock ${ }^{74}$, T. Lazovich ${ }^{57}$, O. Le Dortz ${ }^{80}$, E. Le Guirriec ${ }^{85}$, E. Le Menedeu ${ }^{12}$, M. LeBlanc ${ }^{169}$, T. LeCompte ${ }^{6}$, F. Ledroit-Guillon ${ }^{55}$, C. A. Lee ${ }^{145 a}$, S. C. Lee ${ }^{151}$, L. Lee ${ }^{1}$, G. Lefebvre ${ }^{80}$, M. Lefebvre ${ }^{169}$, F. Legger ${ }^{100}$, C. Leggett ${ }^{15}$, A. Lehan ${ }^{74}$, G. Lehmann Miotto ${ }^{30}$, X. Lei ${ }^{7}$, W. A. Leight ${ }^{29}$, A. Leisos ${ }^{154, x}$, A. G. Leister ${ }^{176}$, M. A. L. Leite ${ }^{24 d}$, R. Leitner ${ }^{129}$, D. Lellouch ${ }^{172}$, B. Lemmer ${ }^{54}$, K. J. C. Leney ${ }^{78}$, T. Lenz ${ }^{21}$, B. Lenzi ${ }^{30}$, R. Leone ${ }^{7}$, S. Leone ${ }^{124 a}$,124b,$\quad$ C. Leonidopoulos ${ }^{46}$, S. Leontsinis ${ }^{10}$, C. Leroy ${ }^{95}$, C. G. Lester ${ }^{28}$, M. Levchenko ${ }^{123}$, J. Levêque ${ }^{5}$, D. Levin ${ }^{89}$, L. J. Levinson ${ }^{172}$, M. Levy ${ }^{18}$, A. Lewis ${ }^{120}$, A. M. Leyko ${ }^{21}$, M. Leyton ${ }^{41}$, B. Li ${ }^{33 b, y}$, H. Li ${ }^{148}$, H. L. Li ${ }^{31}$, L. Li $^{45}$,

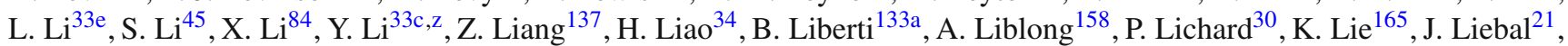
W. Liebig ${ }^{14}$, C. Limbach ${ }^{21}$, A. Limosani ${ }^{150}$, S. C. $\operatorname{Lin}^{151}$,aa , T. H. Lin ${ }^{83}$, F. Linde ${ }^{107}$, B. E. Lindquist ${ }^{148}$, J. T. Linnemann ${ }^{90}$, E. Lipeles ${ }^{122}$, A. Lipniacka14, M. Lisovyi ${ }^{58 b}$, T. M. Liss ${ }^{165}$, D. Lissauer ${ }^{25}$, A. Lister ${ }^{168}$, A. M. Litke ${ }^{137}$, B. Liu ${ }^{151, a b}$,

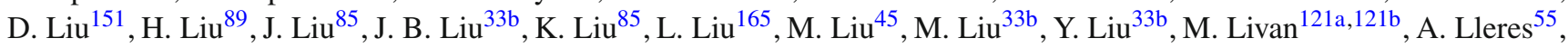
J. Llorente Merino ${ }^{82}$, S. L. Lloyd ${ }^{76}$, F. Lo Sterzo ${ }^{151}$, E. Lobodzinska ${ }^{42}$, P. Loch ${ }^{7}$, W. S. Lockman ${ }^{137}$, F. K. Loebinger ${ }^{84}$, A. E. Loevschall-Jensen ${ }^{36}$, K. M. Loew ${ }^{23}$, A. Loginov ${ }^{176}$, T. Lohse ${ }^{16}$, K. Lohwasser ${ }^{42}$, M. Lokajicek ${ }^{127}$, B. A. Long ${ }^{22}$, J. D. Long ${ }^{165}$, R. E. Long ${ }^{72}$, K. A. Looper ${ }^{111}$, L. Lopes ${ }^{126 a}$, D. Lopez Mateos ${ }^{57}$, B. Lopez Paredes ${ }^{139}$, I. Lopez Paz ${ }^{12}$, J. Lorenz ${ }^{100}$, N. Lorenzo Martinez ${ }^{61}$, M. Losada ${ }^{162}$, P. J. Lösel100, X. Lou ${ }^{33 a}$, A. Lounis ${ }^{117}$, J. Love ${ }^{6}$, P. A. Love ${ }^{72}$, H. Lu ${ }^{60 a}$, N. Lu ${ }^{89}$, H. J. Lubatti ${ }^{138}$, C. Luci ${ }^{132 a, 132 b}$, A. Lucotte ${ }^{55}$, C. Luedtke ${ }^{48}$, F. Luehring ${ }^{61}$, W. Lukas ${ }^{62}$, L. Luminari ${ }^{132 a}$, O. Lundberg ${ }^{146 a}$,146b , B. Lund-Jensen ${ }^{147}$, D. Lynn ${ }^{25}$, R. Lysak ${ }^{127}$, E. Lytken ${ }^{81}, \mathrm{H}_{\text {. Ma }}{ }^{25}$, L. L. Ma ${ }^{33 d}$, G. Maccarrone ${ }^{47}$, A. Macchiolo ${ }^{101}$, C. M. Macdonald ${ }^{139}$, B. Maček ${ }^{75}$, J. Machado Miguens ${ }^{122,126 b}$, D. Macina ${ }^{30}$, D. Madaffari ${ }^{85}$, 
R. Madar ${ }^{34}$, H. J. Maddocks ${ }^{72}$, W. F. Mader ${ }^{44}$, A. Madsen ${ }^{166}$, J. Maeda ${ }^{67}$, S. Maeland ${ }^{14}$, T. Maeno ${ }^{25}$, A. Maevskiy ${ }^{99}$, E. Magradze ${ }^{54}$, K. Mahboubi ${ }^{48}$, J. Mahlstedt ${ }^{107}$, C. Maiani ${ }^{136}$, C. Maidantchik ${ }^{24 a}$, A. A. Maier ${ }^{101}, \quad$ T. Maier ${ }^{100}$, A. Maio ${ }^{126 a, 126 b, 126 d}$, S. Majewski ${ }^{116}$, Y. Makida ${ }^{66}$, N. Makovec ${ }^{117}$, B. Malaescu ${ }^{80}$, Pa. Malecki ${ }^{39}$, V. P. Maleev ${ }^{123}$, F. Malek ${ }^{55}$, U. Mallik ${ }^{63}$, D. Malon ${ }^{6}$, C. Malone ${ }^{143}$, S. Maltezos ${ }^{10}$, V. M. Malyshev ${ }^{109}$, S. Malyukov ${ }^{30}$, J. Mamuzic ${ }^{42}$, G. Mancini ${ }^{47}$, B. Mandelli ${ }^{30}$, L. Mandelli91a, I. Mandićc ${ }^{75}$, R. Mandrysch ${ }^{63}$, J. Maneira ${ }^{126 a, 126 b}$, A. Manfredini ${ }^{101}$, L. Manhaes de Andrade Filho ${ }^{24 b}$, J. Manjarres Ramos ${ }^{159 b}$, A. Mann ${ }^{100}$, A. Manousakis-Katsikakis ${ }^{9}$, B. Mansoulie ${ }^{136}$, R. Mantifel ${ }^{87}$, M. Mantoani ${ }^{54}$, L. Mapelli ${ }^{30}$, L. March ${ }^{145 c}$, G. Marchiori ${ }^{80}$ ， M. Marcisovsky ${ }^{127}$, C. P. Marino ${ }^{169}$, M. Marjanovic ${ }^{13}$, D. E. Marley ${ }^{89}$, F. Marroquim ${ }^{24 a}$, S. P. Marsden ${ }^{84}$, Z. Marshall ${ }^{15}$, L. F. Marti ${ }^{17}$, S. Marti-Garcia ${ }^{167}$, B. Martin ${ }^{90}$, T. A. Martin ${ }^{170}$, V. J. Martin ${ }^{46}$, B. Martin dit Latour ${ }^{14}$, M. Martinez ${ }^{12, o}$, S. Martin-Haugh ${ }^{131}$, V. S. Martoiu ${ }^{26 b}$, A. C. Martyniuk ${ }^{78}$, M. Marx ${ }^{138}$, F. Marzano ${ }^{132 a}$, A. Marzin $^{30}$, L. Masetti ${ }^{83}$, T. Mashimo ${ }^{155}$, R. Mashinistov ${ }^{96}$, J. Masik ${ }^{84}$, A. L. Maslennikov ${ }^{109, c}$, I. Massa ${ }^{20 a, 20 b}$, L. Massa ${ }^{20 a, 20 b}$, P. Mastrandrea ${ }^{5}$, A. Mastroberardino ${ }^{37 a, 37 b}$, T. Masubuchi ${ }^{155}$, P. Mättig ${ }^{175}$ ， J. Mattmann ${ }^{83}$ ，J. Maurer ${ }^{26 b}$ ，S. J. Maxfield ${ }^{74}$ ， D. A. Maximov ${ }^{109, c}$ ， R. Mazini151， S. M. Mazza ${ }^{91 a, 91 b}$,

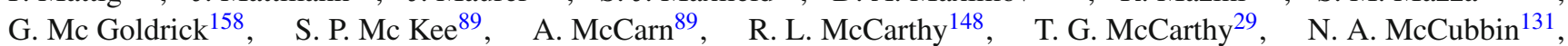
K. W. McFarlane ${ }^{56, *}$, J. A. Mcfayden ${ }^{78}$, G. Mchedlidze ${ }^{54}$, S. J. McMahon ${ }^{131}$, R. A. McPherson ${ }^{169, \mathrm{k}}$, M. Medinnis ${ }^{42}$, S. Meehan ${ }^{145 a}$, S. Mehlhase ${ }^{100}$, A. Mehta ${ }^{74}$, K. Meier ${ }^{58 a}$, C. Meineck ${ }^{100}$, B. Meirose ${ }^{41}$, B. R. Mellado Garcia ${ }^{145 c}$, F. Meloni ${ }^{17}$, A. Mengarelli ${ }^{20 a, 20 b}$, S. Menke ${ }^{101}$, E. Meoni ${ }^{161}$, K. M. Mercurio ${ }^{57}$, S. Mergelmeyer ${ }^{21}$, P. Mermod ${ }^{49}$, L. Merola ${ }^{104 a, 104 b}$,C. Meroni ${ }^{91 a}$, F. S. Merritt ${ }^{31}$, A. Messina ${ }^{132 a, 132 b}$, J. Metcalfe ${ }^{25}$, A. S. Mete ${ }^{163}$, C. Meyer ${ }^{83}$, C. Meyer ${ }^{122}$, J-P. Meyer ${ }^{136}$, J. Meyer ${ }^{107}$, H. Meyer Zu Theenhausen ${ }^{58 a}$, R. P. Middleton ${ }^{131}$, S. Miglioranzi ${ }^{164 a, 164 c}$, L. Mijović ${ }^{21}$, G. Mikenberg ${ }^{172}$, M. Mikestikova ${ }^{127}$, M. Mikuž ${ }^{75}$, M. Milesi ${ }^{88}$, A. Milic ${ }^{30}$, D. W. Miller ${ }^{31}$, C. Mills ${ }^{46}$, A. Milov ${ }^{172}$, D. A. Milstead ${ }^{146 a, 146 b}$, A. A. Minaenko ${ }^{130}$, Y. Minami ${ }^{155}$, I. A. Minashvili65, A. I. Mincer ${ }^{110}$, B. Mindur ${ }^{38 a}$, M. Mineev ${ }^{65}$, Y. Ming ${ }^{173}$, L. M. Mir ${ }^{12}$, K. P. Mistry ${ }^{122}$, T. Mitani ${ }^{171}$, J. Mitrevski ${ }^{100}$, V. A. Mitsou ${ }^{167}$, A. Miucci ${ }^{49}$, P. S. Miyagawa ${ }^{139}$, J. U. Mjörnmark ${ }^{81}$, T. Moa ${ }^{146 a, 146 b}$, K. Mochizuki ${ }^{85}$, S. Mohapatra ${ }^{35}$, W. Mohr ${ }^{48}$, S. Molander ${ }^{146 a, 146 b}$, R. Moles-Valls ${ }^{21}$, R. Monden ${ }^{68}$, K. Mönig ${ }^{42}$, C. Monini ${ }^{55}$, J. Monk ${ }^{36}$, E. Monnier ${ }^{85}$, A. Montalbano ${ }^{148}$, J. Montejo Berlingen ${ }^{12}$, F. Monticellii ${ }^{71}$, S. Monzani ${ }^{132 a, 132 b}$, R. W. Moore ${ }^{3}$, N. Morange ${ }^{117}$, D. Moreno ${ }^{162}$, M. Moreno Llácer ${ }^{54}$, P. Morettini ${ }^{50 a}$, D. Mori ${ }^{142}$, T. Mori ${ }^{155}$, M. Morii ${ }^{57}$, M. Morinaga ${ }^{155}$, V. Morisbak ${ }^{119}$, S. Moritz ${ }^{83}$, A. K. Morley ${ }^{150}$, G. Mornacchi ${ }^{30}$, J. D. Morris ${ }^{76}$, S. S. Mortensen ${ }^{36}$, A. Morton ${ }^{53}$, L. Morvaj ${ }^{103}$, M. Mosidze ${ }^{51 b}$, J. Moss ${ }^{143}$, K. Motohashi ${ }^{157}$, R. Mount ${ }^{143}$, E. Mountricha ${ }^{25}$, S. V. Mouraviev ${ }^{96, *}$, E. J. W. Moyse ${ }^{86}$, S. Muanza ${ }^{85}$, R. D. Mudd ${ }^{18}$, F. Mueller ${ }^{101}$, J. Mueller ${ }^{125}$,

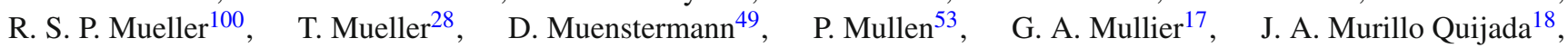

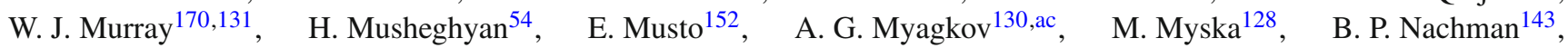
O. Nackenhorst ${ }^{54}$, J. Nadal ${ }^{54}$, K. Nagai ${ }^{120}$, R. Nagai ${ }^{157}$, Y. Nagai ${ }^{85}$, K. Nagano ${ }^{66}$, A. Nagarkar ${ }^{111}$, Y. Nagasaka ${ }^{59}$, K. Nagata ${ }^{160}$, M. Nagel ${ }^{101}$, E. Nagy ${ }^{85}$, A. M. Nairz ${ }^{30}$, Y. Nakahama ${ }^{30}$, K. Nakamura ${ }^{66}$, T. Nakamura ${ }^{155}$, I. Nakano ${ }^{112}$, H. Namasivayam ${ }^{41}$, R. F. Naranjo Garcia ${ }^{42}$, R. Narayan ${ }^{31}$, D. I. Narrias Villar ${ }^{58 a}$, T. Naumann ${ }^{42}$, G. Navarro ${ }^{162}$, R. Nayyar ${ }^{7}$, H. A. Neal ${ }^{89}$, P. Yu. Nechaeva ${ }^{96}$, T. J. Neep ${ }^{84}$, P. D. Nef ${ }^{143}$, A. Negri121a,121b, M. Negrini ${ }^{20 a}$, S. Nektarijevic ${ }^{106}$, C. Nellist ${ }^{117}$, A. Nelson ${ }^{163}$, S. Nemecek ${ }^{127}$, P. Nemethy ${ }^{110}$, A. A. Nepomuceno ${ }^{24 a}$, M. Nessi ${ }^{30, a d}$, M. S. Neubauer ${ }^{165}$, M. Neumann ${ }^{175}$, R. M. Neves ${ }^{110}$, P. Nevski ${ }^{25}$, P. R. Newman ${ }^{18}$, D. H. Nguyen ${ }^{6}$, R. B. Nickerson ${ }^{120}$, R. Nicolaidou ${ }^{136}$, B. Nicquevert ${ }^{30}$, J. Nielsen ${ }^{137}$, N. Nikiforou ${ }^{35}$, A. Nikiforov ${ }^{16}$, V. Nikolaenko ${ }^{130, \text { ac }^{2}}$, I. Nikolic-Audit ${ }^{80}$, K. Nikolopoulos ${ }^{18}$, J. K. Nilsen ${ }^{119}$, P. Nilsson ${ }^{25}$, Y. Ninomiya ${ }^{155}$, A. Nisati ${ }^{132 a}$, R. Nisius ${ }^{101}$, T. Nobe ${ }^{155}$, M. Nomachi ${ }^{118}$, I. Nomidis ${ }^{29}$, T. Nooney ${ }^{76}$, S. Norberg ${ }^{113}$, M. Nordberg ${ }^{30}$, O. Novgorodova ${ }^{44}$, S. Nowak ${ }^{101}$, M. Nozaki ${ }^{66}$, L. Nozka ${ }^{15}$, K. Ntekas ${ }^{10}$, G. Nunes Hanninger ${ }^{88}$, T. Nunnemann ${ }^{100}$, E. Nurse ${ }^{78}$, F. Nuti ${ }^{88}$, B. J. O’Brien ${ }^{46}$, F. O' $\operatorname{grady}^{7}$, D. C. O'Neil ${ }^{142}$, V. O’'Shea ${ }^{53}$, F. G. Oakham ${ }^{29, \text { d }}$, H. Oberlack ${ }^{101}$, T. Obermann ${ }^{21}$, J. Ocariz ${ }^{80}$, A. Ochi ${ }^{67}$, I. Ochoa ${ }^{35}$, J. P. Ochoa-Ricoux ${ }^{32 a}$, S. Oda ${ }^{70}$, S. Odaka ${ }^{66}$, H. Ogren ${ }^{61}$, A. $\mathrm{Oh}^{84}$, S. H. Oh ${ }^{45}$, C. C. $\mathrm{Ohm}^{15}$, H. Ohman ${ }^{166}$, H. Oide ${ }^{30}$, W. Okamura ${ }^{118}$, H. Okawa ${ }^{160}$, Y. Okumura ${ }^{31}$, T. Okuyama ${ }^{66}$, A. Olariu ${ }^{26 b}$, S. A. Olivares Pino ${ }^{46}$, D. Oliveira Damazio ${ }^{25}$, A. Olszewski ${ }^{39}$, J. Olszowska $^{39}$, A. Onofre ${ }^{126 a, 126 e}$, K. Onogi ${ }^{103}$, P. U. E. Onyisi ${ }^{31, s}$, C. J. Oram ${ }^{159 a}$, M. J. Oreglia ${ }^{31}$, Y. Oren ${ }^{153}$, D. Orestano ${ }^{134 a, 134 b}$, N. Orlando ${ }^{154}$, C. Oropeza Barrera ${ }^{53}$, R. S. Orr ${ }^{158}$, B. Osculati ${ }^{50 a, 50 b}$, R. Ospanov ${ }^{84}$, G. Otero y Garzon ${ }^{27}$, H. Otono ${ }^{70}$, M. Ouchrif ${ }^{135 d}$, F. Ould-Saada ${ }^{119}$, A. Ouraou ${ }^{136}$, K. P. Oussoren ${ }^{107}$, Q. Ouyang ${ }^{33 a}$, A. Ovcharova ${ }^{15}$, M. Owen ${ }^{53}$, R. E. Owen ${ }^{18}$, V. E. Ozcan ${ }^{19 a}$, N. Ozturk ${ }^{8}$, K. Pachal ${ }^{142}$, A. Pacheco Pages ${ }^{12}$, C. Padilla Aranda ${ }^{12}$, M. Pagáčová ${ }^{48}$, S. Pagan Griso ${ }^{15}$, E. Paganis ${ }^{139}$, F. Paige ${ }^{25}$, P. Pais ${ }^{86}$, K. Pajchel ${ }^{119}$, G. Palacino ${ }^{159 b}$, S. Palestini ${ }^{30}$, M. Palka ${ }^{38 b}$, D. Pallin ${ }^{34}$, A. Palma ${ }^{126 a, 126 b}$, Y. B. Pan ${ }^{173}$, E. St. Panagiotopoulou ${ }^{10}, \quad$ C. E. Pandini ${ }^{80}$, J. G. Panduro Vazquez ${ }^{77}$, P. Pani ${ }^{146 a}$,146b , S. Panitkin ${ }^{25}$, D. Pantea ${ }^{26 b}$, L. Paolozzi ${ }^{49}$, Th. D. Papadopoulou ${ }^{10}$, K. Papageorgiou ${ }^{154}$, A. Paramonov ${ }^{6}$, D. Paredes Hernandez ${ }^{154}$, M. A. Parker ${ }^{28}$, K. A. Parker ${ }^{139}$, F. Parodi ${ }^{50 a, 50 b}$, J. A. Parsons ${ }^{35}$, U. Parzefall ${ }^{48}$, E. Pasqualucci ${ }^{132 a}$ ， S. Passaggio ${ }^{50 a}$, F. Pastore ${ }^{134 a, 134 b, *}$, Fr. Pastore ${ }^{77}$, G. Pásztor ${ }^{29}$, S. Pataraia ${ }^{175}$, N. D. Patel ${ }^{150}$,

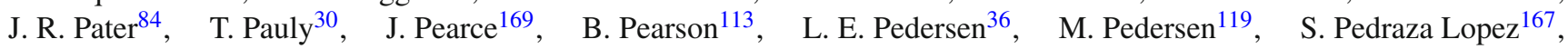
R. Pedro ${ }^{126 a, 126 b}$, S. V. Peleganchuk ${ }^{109, c}$, D. Pelikan ${ }^{166}$, O. Penc ${ }^{127}$, C. Peng ${ }^{33 a}$, H. Peng ${ }^{33 b}$, B. Penning ${ }^{31}$, J. Penwell ${ }^{61}$, 
D. V. Perepelitsa ${ }^{25}$, E. Perez Codina ${ }^{159 a}$, M. T. Pérez García-Estañ ${ }^{167}$, L. Perini ${ }^{91 a}$,91b ${ }^{3}$ H. Pernegger ${ }^{30}$, S. Perrella ${ }^{104 a, 104 b}$, R. Peschke ${ }^{42}$, V. D. Peshekhonov ${ }^{65}$, K. Peters ${ }^{30}$, R. F. Y. Peters ${ }^{84}$, B. A. Petersen ${ }^{30}$, T. C. Petersen ${ }^{36}$, E. Petit ${ }^{42}$, A. Petridis ${ }^{1}$, C. Petridou ${ }^{154}$ ， P. Petroff ${ }^{117}$ ， E. Petrolo ${ }^{132 a}$, F. Petrucci ${ }^{134 a, 134 b}$ ， N. E. Pettersson ${ }^{157}$ ， R. Pezoa ${ }^{32 b}$ ， P. W. Phillips ${ }^{131}$, G. Piacquadio ${ }^{143}$, E. Pianori ${ }^{170}$, A. Picazio ${ }^{49}$, E. Piccaro ${ }^{76}$, M. Piccinini ${ }^{20 a, 20 b}$, M. A. Pickering ${ }^{120}$, R. Piegaia ${ }^{27}$, D. T. Pignotti ${ }^{111}$ ， J. E. Pilcher ${ }^{31}$ ， A. D. Pilkington ${ }^{84}$, A. W. J. Pin ${ }^{84}$, J. Pina ${ }^{126 a, 126 b, 126 d}$, M. Pinamonti ${ }^{164 a, 164 c, a e,}$ J. L. Pinfold ${ }^{3}$, A. Pingel ${ }^{36}$, S. Pires ${ }^{80}, \quad$ H. Pirumov ${ }^{42}$, M. Pitt ${ }^{172}$, C. Pizio ${ }^{91 a, 91 b}, \quad$ L. Plazak ${ }^{144 a}, \quad$ M.-A. Pleier ${ }^{25}$, V. Pleskot ${ }^{129}$ ， E. Plotnikova ${ }^{65}$, P. Plucinski ${ }^{146 a, 146 b}$, D. Pluth ${ }^{64}, \quad$ R. Poettgen ${ }^{146 a, 146 b}, \quad$ L. Poggioli ${ }^{117}, \quad$ D. Pohl ${ }^{21}$, G. Polesello ${ }^{121 a}$, A. Poley ${ }^{42}$, A. Policicchio ${ }^{37 a, 37 b}$, R. Polifka ${ }^{158}$, A. Polini ${ }^{20 a}$, C. S. Pollard ${ }^{53}$, V. Polychronakos ${ }^{25}$, K. Pommès ${ }^{30}$, L. Pontecorvo ${ }^{132 a}$, B. G. Pope ${ }^{90}$, G. A. Popeneciu ${ }^{26 c}$, D. S. Popovic ${ }^{13}$, A. Poppleton ${ }^{30}$, S. Pospisil ${ }^{128}$, K. Potamianos ${ }^{15}$, I. N. Potrap ${ }^{65}$, C. J. Potter ${ }^{149}$, C. T. Potter ${ }^{116}$, G. Poulard ${ }^{30}$, J. Poveda ${ }^{30}$, V. Pozdnyakov ${ }^{65}$,

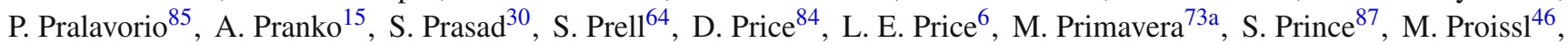
K. Prokofiev ${ }^{60 c}$, F. Prokoshin ${ }^{32 b}$, E. Protopapadaki ${ }^{136}$, S. Protopopescu ${ }^{25}$, J. Proudfoot ${ }^{6}$, M. Przybycien ${ }^{38 a}$, E. Ptacek ${ }^{116}$, D. Puddu 134a,134b , E. Pueschel ${ }^{86}$, D. Puldon ${ }^{148}$, M. Purohit ${ }^{25}$,af, P. Puzo ${ }^{117}$, J. Qian ${ }^{89}$, G. Qin ${ }^{53}$, Y. Qin ${ }^{84}$, A. Quadt ${ }^{54}$, D. R. Quarrie ${ }^{15}$, W. B. Quayle ${ }^{164 a, 164 b}$, M. Queitsch-Maitland ${ }^{84}$, D. Quilty ${ }^{53}$, S. Raddum ${ }^{119}$, V. Radeka ${ }^{25}$, V. Radescu ${ }^{42}$, S. K. Radhakrishnan ${ }^{148}$, P. Radloff ${ }^{16}$, P. Rados ${ }^{88}$, F. Ragusa ${ }^{91 a}$,91b, G. Rahal ${ }^{178}$, S. Rajagopalan ${ }^{25}$, M. Rammensee ${ }^{30}$, C. Rangel-Smith ${ }^{166}$, F. Rauscher ${ }^{100}$ ， S. Rave ${ }^{83}$, T. Ravenscroft ${ }^{53}$ ， M. Raymond ${ }^{30}$ ， A. L. Read ${ }^{119}$, N. P. Readioff ${ }^{74}$, D. M. Rebuzzi ${ }^{121 a, 121 b}$ ，A. Redelbach ${ }^{174}$ ， G. Redlinger ${ }^{25}$ ，R. Reece ${ }^{137}$ ，K. Reeves ${ }^{41}$ ， L. Rehnisch ${ }^{16}$ ， J. Reichert ${ }^{122}$, H. Reisin ${ }^{27}$, C. Rembser $^{30}$, H. $\operatorname{Ren}^{33 a}$, A. Renaud ${ }^{117}$, M. Rescigno ${ }^{132 a}$, S. Resconi ${ }^{91 a}$, O. L. Rezanova ${ }^{109, c}$, P. Reznicek ${ }^{129}$, R. Rezvani ${ }^{95}$ ，R. Richter ${ }^{101}$ ，S. Richter ${ }^{78}$, E. Richter-Was ${ }^{38 b}$ ，O. Ricken ${ }^{21}$ ，M. Ridel ${ }^{80}$ ，P. Rieck ${ }^{16}$ ，C. J. Riegel ${ }^{175}$, J. Rieger ${ }^{54}$, O. Rifki ${ }^{113}$ ， M. Rijssenbeek ${ }^{148}$, A. Rimoldi ${ }^{121 a, 121 b}$ ， L. Rinaldi ${ }^{20 a}$, B. Ristić49, E. Ritsch ${ }^{30}$, I. Riu ${ }^{12}$, F. Rizatdinova ${ }^{114}$ ， E. Rizvi ${ }^{76}$ ， S. H. Robertson ${ }^{87, k}$, A. Robichaud-Veronneau ${ }^{87}$, D. Robinson ${ }^{28}$, J. E. M. Robinson ${ }^{42}$, A. Robson ${ }^{53}$ ， C. Roda ${ }^{124 a, 124 b}$ ，S. Roe ${ }^{30}$ ，O. Røhne ${ }^{119}$ ，A. Romaniouk ${ }^{98}$, M. Romano 20a,20b , S. M. Romano Saez ${ }^{34}$, E. Romero Adam ${ }^{167}$, N. Rompotis ${ }^{138}$, M. Ronzani ${ }^{48}$, L. Roos ${ }^{80}$, E. Ros ${ }^{167}$, S. Rosati ${ }^{132 a}$, K. Rosbach ${ }^{48}$, P. Rose ${ }^{137}$, P. L. Rosendahl ${ }^{14}$, O. Rosenthal ${ }^{141}$, V. Rossetti ${ }^{146 a, 146 b}$, E. Rossi ${ }^{104 a, 104 b}$, L. P. Rossi ${ }^{50 a}$, J. H. N. Rosten ${ }^{28}$, R. Rosten ${ }^{138}$, M. Rotaru ${ }^{26 b}$ ，I. Roth ${ }^{172}$ ，J. Rothberg ${ }^{138}$, D. Rousseau ${ }^{117}$, C. R. Royon ${ }^{136}$, A. Rozanov ${ }^{85}$, Y. Rozen ${ }^{152}$, X. Ruan ${ }^{145 c}$, F. Rubbo ${ }^{143}$, I. Rubinskiy ${ }^{42}$, V. I. Rud ${ }^{99}$, C. Rudolph ${ }^{44}$, M. S. Rudolph ${ }^{158}$, F. Rühr ${ }^{48}$, A. Ruiz-Martinez ${ }^{30}$, Z. Rurikova ${ }^{48}$, N. A. Rusakovich ${ }^{65}$, A. Ruschke ${ }^{100}$, H. L. Russell ${ }^{138}$, J. P. Rutherfoord ${ }^{7}$, N. Ruthmann ${ }^{30}$, Y. F. Ryabov ${ }^{123}$, M. Rybar ${ }^{165}$, G. Rybkin ${ }^{117}$, N. C. Ryder ${ }^{120}$, A. F. Saavedra ${ }^{150}$, G. Sabato ${ }^{107}$, S. Sacerdoti ${ }^{27}$, A. Saddique ${ }^{3}$, H. F-W. Sadrozinski ${ }^{137}$, R. Sadykov ${ }^{65}$, F. Safai Tehrani ${ }^{132 a}$, P. Saha ${ }^{108}$, M. Sahinsoy ${ }^{58 a}$, M. Saimpert ${ }^{136}$, T. Saito ${ }^{155}$, H. Sakamoto ${ }^{155}$, Y. Sakurai ${ }^{171}$, G. Salamanna134a,134b, A. Salamon ${ }^{133 a}$, J. E. Salazar Loyola ${ }^{32 b}$, M. Saleem ${ }^{113}$, D. Salek ${ }^{107}$, P. H. Sales De Bruin ${ }^{138}$,

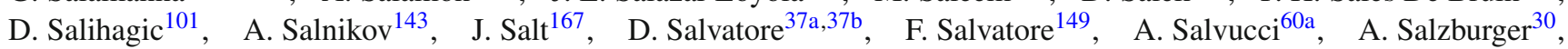
D. Sammel ${ }^{48}$, D. Sampsonidis ${ }^{154}$, A. Sanchez ${ }^{104 a, 104 b}$, J. Sánchez ${ }^{167}, \quad$ V. Sanchez Martinez ${ }^{167}, \quad$ H. Sandaker ${ }^{119}$, R. L. Sandbach $^{76}$, H. G. Sander ${ }^{83}$, M. P. Sanders ${ }^{100}$, M. Sandhoff ${ }^{175}$, C. Sandoval ${ }^{162}$, R. Sandstroem ${ }^{101}$, D. P. C. Sankey ${ }^{131}$, M. Sannino ${ }^{50 a, 50 b}$, A. Sansoni ${ }^{47}$, C. Santoni ${ }^{34}$, R. Santonico ${ }^{133 a, 133 b}$, H. Santos ${ }^{126 a}$, I. Santoyo Castillo ${ }^{149}$, K. Sapp ${ }^{125}$, A. Sapronov ${ }^{65}$, J. G. Saraiva ${ }^{126 a, 126 d}$, B. Sarrazin ${ }^{21}$, O. Sasaki ${ }^{66}$, Y. Sasaki ${ }^{155}$, K. Sato ${ }^{160}$, G. Sauvage ${ }^{5}$ *, E. Sauvan ${ }^{5}$, G. Savage ${ }^{77}$, P. Savard ${ }^{158, d}$, C. Sawyer ${ }^{131}$, L. Sawyer ${ }^{79, n}$, J. Saxon ${ }^{31}$, C. Sbarra ${ }^{20 a}$, A. Sbrizzi ${ }^{20 a, 20 b}$, T. Scanlon ${ }^{78}$, D. A. Scannicchio ${ }^{163}$, M. Scarcella ${ }^{150}$, V. Scarfone ${ }^{37 a, 37 b}$, J. Schaarschmidt ${ }^{172}$, P. Schacht ${ }^{101}$, D. Schaefer ${ }^{30}$, R. Schaefer ${ }^{42}$, J. Schaeffer ${ }^{83}$ ， S. Schaepe ${ }^{21}$ ，S. Schaetzel ${ }^{58 b}$, U. Schäfer ${ }^{83}$ ，A. C. Schaffer ${ }^{117}$ ，D. Schaile ${ }^{100}$, R. D. Schamberger ${ }^{148}$, V. Scharf ${ }^{58 a}$, V. A. Schegelsky ${ }^{123}$, D. Scheirich ${ }^{129}$, M. Schernau ${ }^{163}$, C. Schiavi ${ }^{50 a, 50 b}$, C. Schillo ${ }^{48}$, M. Schioppa ${ }^{37 a, 37 b}$, S. Schlenker ${ }^{30}$, K. Schmieden ${ }^{30}$, C. Schmitt ${ }^{83}$, S. Schmitt ${ }^{58 b}$, S. Schmitt ${ }^{42}$, B. Schneider ${ }^{159 a}$, Y. J. Schnellbach ${ }^{74}$, U. Schnoor ${ }^{44}$, L. Schoeffel ${ }^{136}$, A. Schoening ${ }^{58 b}$, B. D. Schoenrock ${ }^{90}$, E. Schopf ${ }^{21}$, A. L. S. Schorlemmer ${ }^{54}$, M. Schott ${ }^{83}$, D. Schouten ${ }^{159}$, J. Schovancova ${ }^{8}$, S. Schramm ${ }^{49}$, M. Schreyer ${ }^{174}$, N. Schuh ${ }^{83}$, M. J. Schultens ${ }^{21}$, H.-C. Schultz-Coulon ${ }^{58 a}$,

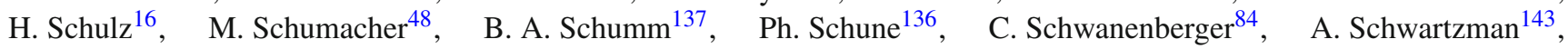
T. A. Schwarz ${ }^{89}$, Ph. Schwegler ${ }^{101}$, H. Schweiger ${ }^{84}$, Ph. Schwemling ${ }^{136}, \quad$ R. Schwienhorst ${ }^{90}$, J. Schwindling ${ }^{136}$, T. Schwindt ${ }^{21}$, F. G. Sciacca ${ }^{17}$, E. Scifo ${ }^{117}$, G. Sciolla ${ }^{23}$, F. Scuri ${ }^{124 a, 124 b}$, F. Scutti ${ }^{21}$, J. Searcy ${ }^{89}$, G. Sedov ${ }^{42}$, E. Sedykh ${ }^{123}$, P. Seema ${ }^{21}$, S. C. Seidel ${ }^{105}$, A. Seiden ${ }^{137}$, F. Seifert ${ }^{128}$, J. M. Seixas ${ }^{24 a}$, G. Sekhniaidze ${ }^{104 a}$, K. Sekhon ${ }^{89}$, S. J. Sekula ${ }^{40}$, D. M. Seliverstov ${ }^{123, *}$, N. Semprini-Cesari ${ }^{20 a, 20 b}$, C. Serfon $^{30}$, L. Serin ${ }^{117}$, L. Serkin ${ }^{164 a, 164 b}$, T. Serre ${ }^{85}$, M. Sessa ${ }^{134 a, 134 b}$, R. Seuster ${ }^{159 a}$, H. Severini ${ }^{113}$, T. Sfiligoj ${ }^{75}$, F. Sforza ${ }^{30}$, A. Sfyrla ${ }^{30}$, E. Shabalina ${ }^{54}$, M. Shamim ${ }^{116}$, L. Y. Shan ${ }^{33 a}$, R. Shang ${ }^{165}$, J. T. Shank ${ }^{22}$, M. Shapiro ${ }^{15}$, P. B. Shatalov ${ }^{97}$, K. Shaw ${ }^{164 a, 164 b}$, S. M. Shaw ${ }^{84}$, A. Shcherbakova ${ }^{146 a, 146 b}$, C. Y. Shehu ${ }^{149}$, P. Sherwood ${ }^{78}$, L. Shi ${ }^{151, a g}$, S. Shimizu ${ }^{67}$, C. O. Shimmin ${ }^{163}$, M. Shimojima ${ }^{102}$, M. Shiyakova ${ }^{65}$, A. Shmeleva ${ }^{96}$, D. Shoaleh Saadi ${ }^{95}$, M. J. Shochet ${ }^{31}$, S. Shojaii ${ }^{11 a, 91 b}$, S. Shrestha ${ }^{111}$, E. Shulga ${ }^{98}$, M. A. Shupe ${ }^{7}$,

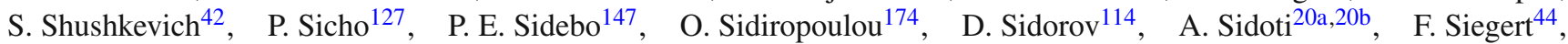
Dj. Sijacki ${ }^{13}$, J. Silva ${ }^{126 a, 126 d}$, Y. Silver ${ }^{153}$, S. B. Silverstein ${ }^{146 a}$, V. Simak ${ }^{128}$, O. Simard ${ }^{5}$, Lj. Simic ${ }^{13}$, S. Simion ${ }^{117}$, 
E. Simioni ${ }^{83}$, B. Simmons ${ }^{78}$, D. Simon ${ }^{34}$, P. Sinervo ${ }^{158}$, N. B. Sinev ${ }^{116}$, M. Sioli ${ }^{20 a, 20 b}$, G. Siragusa ${ }^{174}$, A. N. Sisakyan ${ }^{65, *}$, S. Yu. Sivoklokov ${ }^{99}$ ， J. Sjölin 146a,146b, T. B. Sjursen ${ }^{14}$, M. B. Skinner ${ }^{72}$, H. P. Skottowe ${ }^{57}$, P. Skubic ${ }^{113}$, M. Slater ${ }^{18}$, T. Slavicek ${ }^{128}$, M. Slawinska ${ }^{107}$, K. Sliwa ${ }^{161}$, V. Smakhtin ${ }^{172}$, B. H. Smart ${ }^{46}$, L. Smestad ${ }^{14}$, S. Yu. Smirnov ${ }^{98}$, Y. Smirnov ${ }^{98}$, L. N. Smirnova ${ }^{99, \text { ah }}$, O. Smirnova ${ }^{81}$, M. N. K. Smith ${ }^{35}$, R. W. Smith ${ }^{35}$, M. Smizanska ${ }^{72}$, K. Smolek ${ }^{128}$, A. A. Snesarev ${ }^{96}$, G. Snidero ${ }^{76}$, S. Snyder ${ }^{25}$, R. Sobie ${ }^{169, \mathrm{k}}$, F. Socher ${ }^{44}$, A. Soffer ${ }^{153}$, D. A. Soh ${ }^{151, \text { ag }}$, G. Sokhrannyi ${ }^{75}$, C. A. Solans ${ }^{30}$, M. Solar ${ }^{128}$, J. Solc ${ }^{128}$, E. Yu. Soldatov ${ }^{98}$, U. Soldevila ${ }^{167}$, A. A. Solodkov ${ }^{130}$, A. Soloshenko ${ }^{65}$, O. V. Solovyanov ${ }^{130}$, $\begin{array}{lll}\text { V. Solovyev } & 123\end{array}$ P. Sommer ${ }^{48}$, H. Y. Song ${ }^{33 b, y}$, N. Soni ${ }^{1}$, A. Sood ${ }^{15}$, A. Sopczak ${ }^{128}$, B. Sopko ${ }^{128}$, V. Sopko ${ }^{128}$, V. Sorin ${ }^{12}$, D. Sosa ${ }^{58 b}$, M. Sosebee ${ }^{8}$, C. L. Sotiropoulou ${ }^{124 a, 124 b}$, R. Soualah ${ }^{164 a, 164 c}$, A. M. Soukharev 109,c, D. South ${ }^{42}$, B. C. Sowden ${ }^{77}$, S. Spagnolo ${ }^{73 a}, 73 b$, M. Spalla ${ }^{124 a, 124 b}$, M. Spangenberg ${ }^{170}$, F. Spanò ${ }^{77}$, W. R. Spearman ${ }^{57}$, D. Sperlich ${ }^{16}$, F. Spettel ${ }^{101}$, R. Spighi ${ }^{20 a}$ ，G. Spigo ${ }^{30}$, L. A. Spiller ${ }^{88}$, M. Spousta ${ }^{129}$, R. D. St. Denis ${ }^{53, *}$, A. Stabile ${ }^{91 a}$, S. Staerz ${ }^{44}$, J. Stahlman ${ }^{122}$, R. Stamen ${ }^{58 a}$ ， S. Stamm ${ }^{16}$, E. Stanecka ${ }^{39}$ ， C. Stanescu ${ }^{134 a}$ ， M. Stanescu-Bellu ${ }^{42}$ ， M. M. Stanitzki ${ }^{42}$, S. Stapnes ${ }^{119}$, E. A. Starchenko ${ }^{130}$, J. Stark ${ }^{55}$, P. Staroba ${ }^{127}$, P. Starovoitov ${ }^{58 a}$, R. Staszewski ${ }^{39}$, P. Steinberg ${ }^{25}$, B. Stelzer ${ }^{142}$, H. J. Stelzer ${ }^{30}$, O. Stelzer-Chilton ${ }^{159 a}$, H. Stenzel ${ }^{52}$, G. A. Stewart ${ }^{53}$, J. A. Stillings ${ }^{21}$, M. C. Stockton ${ }^{87}$, M. Stoebe ${ }^{87}$, G. Stoicea ${ }^{26 b}$ ， P. Stolte ${ }^{54}$ ， S. Stonjek ${ }^{101}$ ， A. R. Stradling ${ }^{8}$ ，A. Straessner ${ }^{44}$ ， M. E. Stramaglia ${ }^{17}$ ， J. Strandberg ${ }^{147}$, S. Strandberg ${ }^{146 a}$,146b , A. Strandlie ${ }^{119}$, E. Strauss ${ }^{143}$, M. Strauss ${ }^{113}$, P. Strizenec ${ }^{144 b}$, R. Ströhmer ${ }^{174}$, D. M. Strom ${ }^{116}$, R. Stroynowski ${ }^{40}$, A. Strubig ${ }^{106}$, S. A. Stucci ${ }^{17}$, B. Stugu ${ }^{14}$, N. A. Styles ${ }^{42}$, D. Su ${ }^{143}$, J. Su ${ }^{125}$, R. Subramaniam ${ }^{79}$, A. Succurro ${ }^{12}$, Y. Sugaya ${ }^{118}$, M. Suk ${ }^{128}$, V. V. Sulin ${ }^{96}$, S. Sultansoy ${ }^{4 c}$, T. Sumida ${ }^{68}$, S. Sun $^{57}$, X. Sun ${ }^{33 a}$, J. E. Sundermann ${ }^{48}$, K. Suruliz ${ }^{149}$, G. Susinno ${ }^{37 a, 37 b}$ M. R. Sutton ${ }^{149}$, S. Suzuki ${ }^{66}, \quad$ M. Svatos ${ }^{127}$, M. Swiatlowski ${ }^{143}$, I. Sykora ${ }^{144 a}$, T. Sykora ${ }^{129}$, D. Ta ${ }^{48}$, C. Taccini1 ${ }^{134 a, 134 b}$, K. Tackmann ${ }^{42}$, J. Taenzer ${ }^{158}$, A. Taffard ${ }^{163}$, R. Tafirout ${ }^{159 a}$, N. Taiblum ${ }^{153}$, H. Takai ${ }^{25}$, R. Takashima ${ }^{69}$, H. Takeda ${ }^{67}$, T. Takeshita ${ }^{140}$, Y. Takubo ${ }^{66}$, M. Talby ${ }^{85}$, A. A. Talysheve ${ }^{109, c}$, J. Y. C. Tam ${ }^{174}$, K. G. $\operatorname{Tan}^{88}$, J. Tanaka ${ }^{155}$, R. Tanaka ${ }^{117}, \quad$ S. Tanaka ${ }^{66}, \quad$ B. B. Tannenwald ${ }^{111}, \quad$ N. Tannoury ${ }^{21}, \quad$ S. Tapia Araya ${ }^{32 b}$, S. Tapprogge ${ }^{83}$, S. Tarem ${ }^{152}$, F. Tarrade ${ }^{29}$, G. F. Tartarelli ${ }^{91 a}$, P. Tas ${ }^{129}$, M. Tasevsky ${ }^{127}$, T. Tashiro ${ }^{68}$, E. Tassi ${ }^{37 a, 37 b}$,

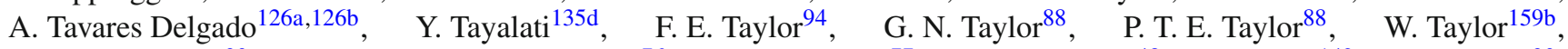
F. A. Teischinger ${ }^{30}$, M. Teixeira Dias Castanheira ${ }^{76}$, P. Teixeira-Dias ${ }^{77}$, K. K. Temming ${ }^{48}$, D. Temple ${ }^{142}$, H. Ten Kate ${ }^{30}$, P. K. Teng ${ }^{151}$, J. J. Teoh ${ }^{118}$, F. Tepel ${ }^{175}$, S. Terada ${ }^{66}$, K. Terashi ${ }^{155}$, J. Terron ${ }^{82}$, S. Terzo ${ }^{101}$, M. Testa ${ }^{47}$, R. J. Teuscher ${ }^{158, k}$, T. Theveneaux-Pelzer ${ }^{34}$, J. P. Thomas ${ }^{18}$, J. Thomas-Wilsker ${ }^{77}$, E. N. Thompson ${ }^{35}$, P. D. Thompson ${ }^{18}$, R. J. Thompson ${ }^{84}$, A. S. Thompson ${ }^{53}$, L. A. Thomsen ${ }^{176}$, E. Thomson ${ }^{122}$, M. Thomson ${ }^{28}$, R. P. Thun ${ }^{89, *}$, M. J. Tibbetts ${ }^{15}$, R. E. Ticse Torres ${ }^{85}$, V. O. Tikhomirov ${ }^{96, a i}$, Yu. A. Tikhonov ${ }^{109, c}, \quad$ S. Timoshenko ${ }^{98}, \quad$ E. Tiouchichine ${ }^{85}, \quad$ P. Tipton ${ }^{176}, \quad$ S. Tisserant ${ }^{85}$, K. Todome ${ }^{157}$, T. Todorov ${ }^{5, *}$, S. Todorova-Nova ${ }^{129}$, J. Tojo ${ }^{70}$, S. Tokár ${ }^{144 a}$, K. Tokushuku ${ }^{66}$, K. Tollefson ${ }^{90}$, E. Tolley ${ }^{57}$, L. Tomlinson ${ }^{84}$, M. Tomoto ${ }^{103}$, L. Tompkins ${ }^{143, \text { aj }}$, K. Toms ${ }^{105}$, E. Torrence ${ }^{116}$, H. Torres $^{142}$, E. Torró Pastor ${ }^{138}$, J. Toth $^{85}$,ak, F. Touchard ${ }^{85}$, D. R. Tovey ${ }^{139}$, T. Trefzger ${ }^{174}$, L. Tremblet $^{30}$, A. Tricoli $^{30}$, I. M. Trigger ${ }^{159 a}$, S. Trincaz-Duvoid ${ }^{80}$, M. F. Tripiana ${ }^{12}$, W. Trischuk ${ }^{158}$, B. Trocmé55, C. Troncon ${ }^{91 a}$, M. Trottier-McDonald ${ }^{15}$, M. Trovatelli $^{169}$, L. Truong $^{164 a, 164 c}$, M. Trzebinski ${ }^{39}$, A. Trzupek ${ }^{39}$, C. Tsarouchas ${ }^{30}$, J. C-L. Tseng ${ }^{120}$, P. V. Tsiareshka ${ }^{92}$, D. Tsionou ${ }^{154}$, G. Tsipolitis ${ }^{10}$, N. Tsirintanis ${ }^{9}$, S. Tsiskaridze ${ }^{12}$, V. Tsiskaridze ${ }^{48}$, E. G. Tskhadadze ${ }^{51 a}$, K. M. Tsui ${ }^{60 a}$, I. I. Tsukerman ${ }^{97}$, V. Tsulaia ${ }^{15}$,

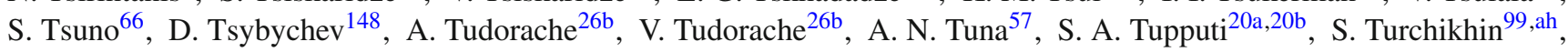
D. Turecek ${ }^{128}$, R. Turra ${ }^{91 a, 91 b}$, A. J. Turvey ${ }^{40}$, P. M. Tuts ${ }^{35}$, A. Tykhonov ${ }^{49}$, M. Tylmad ${ }^{146 a, 146 b}$, M. Tyndel ${ }^{131}$, I. Ueda ${ }^{155}$, R. Ueno ${ }^{29}$, M. Ughetto ${ }^{146 a, 146 b}$, M. Ugland ${ }^{14}$, F. Ukegawa ${ }^{160}$, G. Unal ${ }^{30}$, A. Undrus ${ }^{25}$, G. Unel ${ }^{163}$, F. C. Ungaro ${ }^{48}$, Y. Unno ${ }^{66}$, C. Unverdorben ${ }^{100}$, J. Urban ${ }^{144 b}$, P. Urquijo ${ }^{88}$, P. Urrejola ${ }^{83}$, G. Usai ${ }^{8}$, A. Usanova ${ }^{62}$, L. Vacavant ${ }^{85}$, V. Vacek ${ }^{128}$,

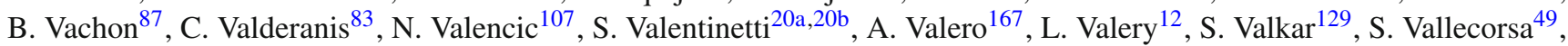

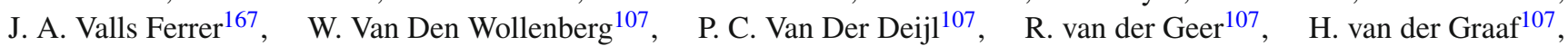
N. van Eldik $^{152}$, P. van Gemmeren ${ }^{6}$, J. Van Nieuwkoop ${ }^{142}$, I. van Vulpen ${ }^{107}$, M. C. van Woerden ${ }^{30}$, M. Vanadia ${ }^{132 a, 132 b}$, W. Vandelli ${ }^{30}$, R. Vanguri ${ }^{122}$, A. Vaniachine ${ }^{6}$, F. Vannucci ${ }^{80}$, G. Vardanyan ${ }^{177}$, R. Vari ${ }^{132 a}$, E. W. Varnes ${ }^{7}$, T. $\operatorname{Varol}^{40}$, D. Varouchas ${ }^{80}$, A. Vartapetian ${ }^{8}$, K. E. Varvell ${ }^{150}$, F. Vazeille ${ }^{34}$, T. Vazquez Schroeder ${ }^{87}$, J. Veatch ${ }^{7}$, L. M. Veloce ${ }^{158}$,

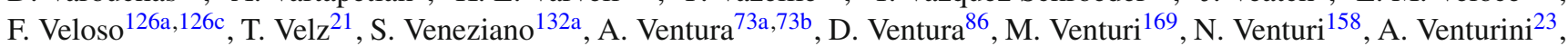
V. Vercesii121a，M. Verducci ${ }^{132 a, 132 b}$ ，W. Verkerke ${ }^{107}$, J. C. Vermeulen ${ }^{107}$, A. Vest ${ }^{44}$, M. C. Vetterli ${ }^{142, d}$, O. Viazlo ${ }^{81}$, I. Vichou ${ }^{165}$, T. Vickey ${ }^{139}$, O. E. Vickey Boeriu ${ }^{139}$, G. H. A. Viehhauser ${ }^{120}$, S. Viel ${ }^{15}$, R. Vigne ${ }^{62}$, M. Villa ${ }^{20 a, 20 b}$,

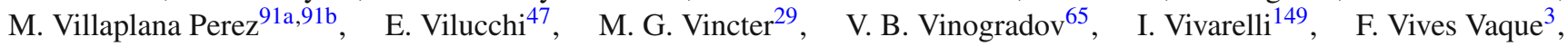
S. Vlachos ${ }^{10}$, D. Vladoiu ${ }^{100}$, M. Vlasak ${ }^{128}$, M. Vogel ${ }^{32 a}$, P. Vokac ${ }^{128}$, G. Volpi ${ }^{124 a, 124 b}$, M. Volpi ${ }^{88}$, H. von der Schmitt ${ }^{101}$, H. von Radziewski ${ }^{48}$, E. von Toerne ${ }^{21}$, V. Vorobel ${ }^{129}$, K. Vorobev ${ }^{98}$, M. Vos $^{167}$, R. Voss ${ }^{30}$, J. H. Vossebeld ${ }^{74}$, N. Vranjes ${ }^{13}$, M. Vranjes Milosavljevic ${ }^{13}$, V. Vrba ${ }^{127}$, M. Vreeswijk ${ }^{107}$, R. Vuillermet ${ }^{30}$, I. Vukotic ${ }^{31}$, Z. Vykydal ${ }^{128}$, P. Wagner ${ }^{21}$, W. Wagner ${ }^{175}$, H. Wahlberg ${ }^{71}$, S. Wahrmund ${ }^{44}$, J. Wakabayashi ${ }^{103}$, J. Walder ${ }^{72}$, R. Walker ${ }^{100}$, W. Walkowiak ${ }^{141}$, C. Wang ${ }^{151}$, F. Wang ${ }^{173}$, H. Wang ${ }^{15}$, H. Wang ${ }^{40}$, J. Wang ${ }^{42}$, J. Wang ${ }^{150}$, K. Wang ${ }^{87}$, R. Wang ${ }^{6}$, S. M. Wang ${ }^{151}$, T. Wang ${ }^{21}$, T. Wang ${ }^{35}$, X. Wang ${ }^{176}$, C. Wanotayaroj ${ }^{116}$, A. Warburton ${ }^{87}$, C. P. Ward ${ }^{28}$, D. R. Wardrope ${ }^{78}$, A. Washbrook ${ }^{46}$, 
C. Wasicki ${ }^{42}$, P. M. Watkins ${ }^{18}$, A. T. Watson ${ }^{18}$, I. J. Watson ${ }^{150}$, M. F. Watson ${ }^{18}$, G. Watts ${ }^{138}$, S. Watts ${ }^{84}$, B. M. Waugh ${ }^{78}$, S. Webb ${ }^{84}$, M. S. Weber ${ }^{17}$, S. W. Weber ${ }^{174}$, J. S. Webster ${ }^{31}$, A. R. Weidberg ${ }^{120}$, B. Weinert ${ }^{61}$, J. Weingarten ${ }^{54}$, C. Weiser ${ }^{48}$, H. Weits ${ }^{107}$, P. S. Wells ${ }^{30}$, T. Wenaus ${ }^{25}$, T. Wengler ${ }^{30}$, S. Wenig ${ }^{30}$, N. Wermes ${ }^{21}$, M. Werner ${ }^{48}$, P. Werner ${ }^{30}$, M. Wessels ${ }^{58 a}$, J. Wetter ${ }^{161}$, K. Whalen ${ }^{116}$, A. M. Wharton ${ }^{72}$, A. White ${ }^{8}$, M. J. White ${ }^{1}$, R. White ${ }^{32 b}$, S. White ${ }^{124 a, 124 b}$, D. Whiteson ${ }^{163}$, F. J. Wickens ${ }^{131}$, W. Wiedenmann ${ }^{173}$, M. Wielers ${ }^{131}$, P. Wienemann ${ }^{21}$, C. Wiglesworth ${ }^{36}$, L. A. M. Wiik-Fuchs ${ }^{21}$, A. Wildauer ${ }^{101}$, H. G. Wilkens ${ }^{30}$, H. H. Williams ${ }^{122}$, S. Williams ${ }^{107}$, C. Willis ${ }^{90}$, S. Willocq ${ }^{86}$, A. Wilson ${ }^{89}$, J. A. Wilson ${ }^{18}$, I. Wingerter-Seez ${ }^{5}$, F. Winklmeier ${ }^{116}$, B. T. Winter ${ }^{21}$, M. Wittgen ${ }^{143}$, J. Wittkowski ${ }^{100}$, S. J. Wollstadt ${ }^{83}$, M. W. Wolter ${ }^{39}$, H. Wolters ${ }^{126 a, 126 c}$, B. K. Wosiek ${ }^{39}$, J. Wotschack ${ }^{30}$, M. J. Woudstra ${ }^{84}$, K. W. Wozniak ${ }^{39}$, M. Wu ${ }^{55}$, M. Wu ${ }^{31}$, S. L. Wu ${ }^{173}$, X. Wu ${ }^{49}$, Y. Wu ${ }^{89}$, T. R. Wyatt ${ }^{84}$, B. M. Wynne ${ }^{46}$, S. Xella ${ }^{36}$, D. Xu ${ }^{33 a}$, L. Xu ${ }^{25}$, B. Yabsley ${ }^{150}, \quad$ S. Yacoob ${ }^{145 a}$, R. Yakabe ${ }^{67}$, M. Yamada ${ }^{66}$, D. Yamaguchi ${ }^{157}$, Y. Yamaguchi ${ }^{118}$, A. Yamamoto ${ }^{66}$, S. Yamamoto ${ }^{155}$, T. Yamanaka ${ }^{155}$, K. Yamauchi ${ }^{103}$, Y. Yamazaki ${ }^{67}$, Z. Yan ${ }^{22}$, H. Yang ${ }^{33 e}$, H. Yang ${ }^{173}$, Y. Yang ${ }^{151}$, W-M. Yao ${ }^{15}$, Y. C. Yap ${ }^{80}$, Y. Yasu ${ }^{66}$, E. Yatsenko ${ }^{5}$, K. H. Yau Wong ${ }^{21}$, J. Ye ${ }^{40}$, S. Ye ${ }^{25}$, I. Yeletskikh ${ }^{65}$, A. L. Yen ${ }^{57}$, E. Yildirim ${ }^{42}$, K. Yorita ${ }^{171}$, R. Yoshida ${ }^{6}$, K. Yoshihara ${ }^{122}$, C. Young ${ }^{143}$, C. J. S. Young ${ }^{30}$, S. Youssef ${ }^{22}$, D. R. Yu ${ }^{15}$, J. Yu ${ }^{8}$, J. M. Yu ${ }^{89}$, J. Yu ${ }^{114}$, L. Yuan ${ }^{67}$, S. P. Y. Yuen ${ }^{21}$, A. Yurkewicz ${ }^{108}$, I. Yusuff ${ }^{28, a l}$, B. Zabinski ${ }^{39}$, R. Zaidan ${ }^{63}$, A. M. Zaitsev ${ }^{130, a c}$, J. Zalieckas ${ }^{14}$, A. Zaman ${ }^{148}$, S. Zambito ${ }^{57}$, L. Zanello ${ }^{132 a, 132 b}$, D. Zanzi ${ }^{88}$, C. Zeitnitz ${ }^{175}$, M. Zeman ${ }^{128}$, A. Zemla ${ }^{38 a}$, Q. Zeng ${ }^{143}$, K. Zengel ${ }^{23}$, O. Zenin ${ }^{130}$, T. Ženišs ${ }^{144 a}$, D. Zerwas ${ }^{117}$, D. Zhang ${ }^{89}$, F. Zhang ${ }^{173}$, G. Zhang ${ }^{33 b}$, H. Zhang ${ }^{33 c}$, J. Zhang ${ }^{6}$, L. Zhang ${ }^{48}$, R. Zhang ${ }^{33 b, i}$, X. Zhang ${ }^{33 d}$, Z. Zhang ${ }^{117}$, X. Zhao ${ }^{40}$, Y. Zhao ${ }^{33 d, 117}$, Z. Zhao $^{33 b}$, A. Zhemchugov ${ }^{65}$, J. Zhong ${ }^{120}$, B. Zhou ${ }^{89}$, C. Zhou ${ }^{45}$, L. Zhou ${ }^{35}$, L. Zhou ${ }^{40}$, M. Zhou ${ }^{148}$, N. Zhou ${ }^{33 f}$, C. G. Zhu ${ }^{33 d}$, H. Zhu ${ }^{33 a}$, J. Zhu ${ }^{89}$, Y. Zhu ${ }^{33 b}$, X. Zhuang ${ }^{33 a}$, K. Zhukov ${ }^{96}$, A. Zibell ${ }^{174}$, D. Zieminska ${ }^{61}$, N. I. Zimine ${ }^{65}$, C. Zimmermann ${ }^{83}$ ， S. Zimmermann ${ }^{48}$ ， Z. Zinonos ${ }^{54}$, M. Zinser ${ }^{83}$, M. Ziolkowski ${ }^{141}$, L. Živković ${ }^{13}$, G. Zobernig ${ }^{173}$, A. Zoccoli ${ }^{20 a, 20 b}$, M. zur Nedden ${ }^{16}$, G. Zurzolo ${ }^{104 a, 104 b}$, L. Zwalinski ${ }^{30}$

${ }^{1}$ Department of Physics, University of Adelaide, Adelaide, Australia

${ }^{2}$ Physics Department, SUNY Albany, Albany, NY, USA

${ }^{3}$ Department of Physics, University of Alberta, Edmonton, AB, Canada

4 (a) Department of Physics, Ankara University, Ankara, Turkey; ${ }^{(b)}$ Istanbul Aydin University, Istanbul,

Turkey; ${ }^{(c)}$ Division of Physics, TOBB University of Economics and Technology, Ankara, Turkey

${ }^{5}$ LAPP, CNRS/IN2P3 and Université Savoie Mont Blanc, Annecy-le-Vieux, France

${ }^{6}$ High Energy Physics Division, Argonne National Laboratory, Argonne, IL, USA

${ }^{7}$ Department of Physics, University of Arizona, Tucson, AZ, USA

${ }^{8}$ Department of Physics, The University of Texas at Arlington, Arlington, TX, USA

${ }^{9}$ Physics Department, University of Athens, Athens, Greece

${ }^{10}$ Physics Department, National Technical University of Athens, Zografou, Greece

${ }^{11}$ Institute of Physics, Azerbaijan Academy of Sciences, Baku, Azerbaijan

${ }^{12}$ Institut de Física d'Altes Energies and Departament de Física de la Universitat Autònoma de Barcelona, Barcelona, Spain

${ }^{13}$ Institute of Physics, University of Belgrade, Belgrade, Serbia

${ }^{14}$ Department for Physics and Technology, University of Bergen, Bergen, Norway

${ }^{15}$ Physics Division, Lawrence Berkeley National Laboratory and University of California, Berkeley, CA, USA

${ }^{16}$ Department of Physics, Humboldt University, Berlin, Germany

${ }^{17}$ Albert Einstein Center for Fundamental Physics and Laboratory for High Energy Physics, University of Bern, Bern, Switzerland

${ }^{18}$ School of Physics and Astronomy, University of Birmingham, Birmingham, UK

19 (a) Department of Physics, Bogazici University, Istanbul, Turkey; ${ }^{(b)}$ Department of Physics Engineering, Gaziantep University, Gaziantep, Turkey; ${ }^{(\mathrm{c})}$ Department of Physics, Dogus University, Gaziantep, Turkey

20 (a) INFN Sezione di Bologna, Bologna, Italy; ${ }^{(b)}$ Dipartimento di Fisica e Astronomia, Università di Bologna, Bologna, Italy

${ }^{21}$ Physikalisches Institut, University of Bonn, Bonn, Germany

${ }^{22}$ Department of Physics, Boston University, Boston, MA, USA

${ }^{23}$ Department of Physics, Brandeis University, Waltham, MA, USA

24 (a) Universidade Federal do Rio De Janeiro COPPE/EE/IF, Rio de Janeiro, Brazil; ${ }^{(b)}$ Electrical Circuits Department, Federal University of Juiz de Fora (UFJF), Juiz de Fora, Brazil; ${ }^{(c)}$ Federal University of Sao Joao del Rei (UFSJ), Sao Joao del Rei, Brazil; ${ }^{(d)}$ Instituto de Fisica, Universidade de Sao Paulo, São Paulo, Brazil

25 Physics Department, Brookhaven National Laboratory, Upton, NY, USA 
26 (a) Transilvania University of Brasov, Brasov, Romania; (b) National Institute of Physics and Nuclear Engineering,

Bucharest, Romania; ${ }^{(c)}$ Physics Department, National Institute for Research and Development of Isotopic and Molecular Technologies, Cluj Napoca, Romania; ${ }^{(d)}$ University Politehnica Bucharest, Bucharest, Romania; ${ }^{(e)}$ West University in

Timisoara, Timisoara, Romania

${ }^{27}$ Departamento de Física, Universidad de Buenos Aires, Buenos Aires, Argentina

${ }^{28}$ Cavendish Laboratory, University of Cambridge, Cambridge, UK

${ }^{29}$ Department of Physics, Carleton University, Ottawa, ON, Canada

${ }^{30}$ CERN, Geneva, Switzerland

${ }^{31}$ Enrico Fermi Institute, University of Chicago, Chicago, IL, USA

32 (a) Departamento de Física, Pontificia Universidad Católica de Chile, Santiago, Chile; ${ }^{(b)}$ Departamento de Física, Universidad Técnica Federico Santa María, Valparaiso, Chile

33 (a) Institute of High Energy Physics, Chinese Academy of Sciences, Beijing, China; ${ }^{(b)}$ Department of Modern Physics, University of Science and Technology of China, Hefei, Anhui, China; ${ }^{\left({ }^{c}\right)}$ Department of Physics, Nanjing University, Nanjing, Jiangsu, China; ${ }^{(d)}$ School of Physics, Shandong University, Shandong, China; ${ }^{\left({ }^{e}\right)}$ Shanghai Key Laboratory for Particle Physics and Cosmology, Department of Physics and Astronomy, Shanghai Jiao Tong University, Shanghai,

China; ${ }^{(f)}$ Physics Department, Tsinghua University, Beijing 100084, China

${ }^{34}$ Laboratoire de Physique Corpusculaire, Clermont Université and Université Blaise Pascal and CNRS/IN2P3,

Clermont-Ferrand, France

${ }^{35}$ Nevis Laboratory, Columbia University, Irvington, NY, USA

${ }^{36}$ Niels Bohr Institute, University of Copenhagen, Copenhagen, Denmark

37 (a) INFN Gruppo Collegato di Cosenza, Laboratori Nazionali di Frascati, Frascati, Italy; ${ }^{(b)}$ Dipartimento di Fisica,

Università della Calabria, Rende, Italy

38 (a) AGH University of Science and Technology, Faculty of Physics and Applied Computer Science, Kraków,

Poland; ${ }^{(b)}$ Marian Smoluchowski Institute of Physics, Jagiellonian University, Kraków, Poland

${ }^{39}$ Institute of Nuclear Physics, Polish Academy of Sciences, Kraków, Poland

${ }^{40}$ Physics Department, Southern Methodist University, Dallas, TX, USA

${ }^{41}$ Physics Department, University of Texas at Dallas, Richardson, TX, USA

42 DESY, Hamburg, Zeuthen, Germany

${ }^{43}$ Institut für Experimentelle Physik IV, Technische Universität Dortmund, Dortmund, Germany

${ }^{44}$ Institut für Kern- und Teilchenphysik, Technische Universität Dresden, Dresden, Germany

${ }^{45}$ Department of Physics, Duke University, Durham, NC, USA

${ }^{46}$ SUPA-School of Physics and Astronomy, University of Edinburgh, Edinburgh, UK

${ }^{47}$ INFN Laboratori Nazionali di Frascati, Frascati, Italy

${ }^{48}$ Fakultät für Mathematik und Physik, Albert-Ludwigs-Universität, Freiburg, Germany

${ }^{49}$ Section de Physique, Université de Genève, Geneva, Switzerland

50 (a) INFN Sezione di Genova, Genoa, Italy; ${ }^{(b)}$ Dipartimento di Fisica, Università di Genova, Genoa, Italy

51 (a) E. Andronikashvili Institute of Physics, Iv. Javakhishvili Tbilisi State University, Tbilisi, Georgia; ${ }^{(b)}$ High Energy

Physics Institute, Tbilisi State University, Tbilisi, Georgia

52 II Physikalisches Institut, Justus-Liebig-Universität Giessen, Giessen, Germany

${ }^{53}$ SUPA-School of Physics and Astronomy, University of Glasgow, Glasgow, UK

${ }^{54}$ II Physikalisches Institut, Georg-August-Universität, Göttingen, Germany

${ }^{55}$ Laboratoire de Physique Subatomique et de Cosmologie, Université Grenoble-Alpes, CNRS/IN2P3, Grenoble, France

${ }^{56}$ Department of Physics, Hampton University, Hampton, VA, USA

${ }^{57}$ Laboratory for Particle Physics and Cosmology, Harvard University, Cambridge, MA, USA

58 (a) Kirchhoff-Institut für Physik, Ruprecht-Karls-Universität Heidelberg, Heidelberg, Germany; ${ }^{(b)}$ Physikalisches Institut, Ruprecht-Karls-Universität Heidelberg, Heidelberg, Germany; ${ }^{(c)}$ ZITI Institut für technische Informatik,

Ruprecht-Karls-Universität Heidelberg, Mannheim, Germany

${ }^{59}$ Faculty of Applied Information Science, Hiroshima Institute of Technology, Hiroshima, Japan

60 (a) Department of Physics, The Chinese University of Hong Kong, Shatin, N.T., Hong Kong; ${ }^{\left({ }^{b}\right)}$ Department of Physics,

The University of Hong Kong, Pokfulam, Hong Kong; ${ }^{(c)}$ Department of Physics, The Hong Kong University of Science and Technology, Clear Water Bay, Kowloon, Hong Kong, China

${ }^{61}$ Department of Physics, Indiana University, Bloomington, IN, USA

${ }^{62}$ Institut für Astro- und Teilchenphysik, Leopold-Franzens-Universität, Innsbruck, Austria 
${ }^{63}$ University of Iowa, Iowa City, IA, USA

${ }^{64}$ Department of Physics and Astronomy, Iowa State University, Ames, IA, USA

65 Joint Institute for Nuclear Research, JINR Dubna, Dubna, Russia

${ }^{66}$ KEK, High Energy Accelerator Research Organization, Tsukuba, Japan

${ }^{67}$ Graduate School of Science, Kobe University, Kobe, Japan

${ }^{68}$ Faculty of Science, Kyoto University, Kyoto, Japan

${ }^{69}$ Kyoto University of Education, Kyoto, Japan

70 Department of Physics, Kyushu University, Fukuoka, Japan

${ }^{71}$ Instituto de Física La Plata, Universidad Nacional de La Plata and CONICET, La Plata, Argentina

72 Physics Department, Lancaster University, Lancaster, UK

73 (a) INFN Sezione di Lecce, Lecce, Italy; ${ }^{\text {(b) }}$ Dipartimento di Matematica e Fisica, Università del Salento, Lecce, Italy

74 Oliver Lodge Laboratory, University of Liverpool, Liverpool, UK

75 Department of Physics, Jožef Stefan Institute and University of Ljubljana, Ljubljana, Slovenia

${ }^{76}$ School of Physics and Astronomy, Queen Mary University of London, London, UK

77 Department of Physics, Royal Holloway University of London, Surrey, UK

${ }^{78}$ Department of Physics and Astronomy, University College London, London, UK

${ }^{79}$ Louisiana Tech University, Ruston, LA, USA

${ }^{80}$ Laboratoire de Physique Nucléaire et de Hautes Energies, UPMC and Université Paris-Diderot and CNRS/IN2P3, Paris,

France

${ }^{81}$ Fysiska institutionen, Lunds universitet, Lund, Sweden

82 Departamento de Fisica Teorica C-15, Universidad Autonoma de Madrid, Madrid, Spain

${ }^{83}$ Institut für Physik, Universität Mainz, Mainz, Germany

${ }^{84}$ School of Physics and Astronomy, University of Manchester, Manchester, UK

${ }^{85}$ CPPM, Aix-Marseille Université and CNRS/IN2P3, Marseille, France

${ }^{86}$ Department of Physics, University of Massachusetts, Amherst, MA, USA

${ }^{87}$ Department of Physics, McGill University, Montreal, QC, Canada

${ }^{88}$ School of Physics, University of Melbourne, Melbourne, VIC, Australia

${ }^{89}$ Department of Physics, The University of Michigan, Ann Arbor, MI, USA

${ }^{90}$ Department of Physics and Astronomy, Michigan State University, East Lansing, MI, USA

91 (a) INFN Sezione di Milano, Milan, Italy; ${ }^{(b)}$ Dipartimento di Fisica, Università di Milano, Milan, Italy

92 B.I. Stepanov Institute of Physics, National Academy of Sciences of Belarus, Minsk, Republic of Belarus

${ }^{93}$ National Scientific and Educational Centre for Particle and High Energy Physics, Minsk, Republic of Belarus

${ }^{94}$ Department of Physics, Massachusetts Institute of Technology, Cambridge, MA, USA

${ }^{95}$ Group of Particle Physics, University of Montreal, Montreal, QC, Canada

${ }^{96}$ P.N. Lebedev Institute of Physics, Academy of Sciences, Moscow, Russia

${ }^{97}$ Institute for Theoretical and Experimental Physics (ITEP), Moscow, Russia

${ }^{98}$ National Research Nuclear University MEPhI, Moscow, Russia

${ }^{99}$ D.V. Skobeltsyn Institute of Nuclear Physics, M.V. Lomonosov Moscow State University, Moscow, Russia

${ }^{100}$ Fakultät für Physik, Ludwig-Maximilians-Universität München, Munich, Germany

${ }^{101}$ Max-Planck-Institut für Physik (Werner-Heisenberg-Institut), Munich, Germany

102 Nagasaki Institute of Applied Science, Nagasaki, Japan

103 Graduate School of Science and Kobayashi-Maskawa Institute, Nagoya University, Nagoya, Japan

104 (a) INFN Sezione di Napoli, Naples, Italy; ${ }^{(b)}$ Dipartimento di Fisica, Università di Napoli, Naples, Italy

105 Department of Physics and Astronomy, University of New Mexico, Albuquerque, NM, USA

${ }^{106}$ Institute for Mathematics, Astrophysics and Particle Physics, Radboud University Nijmegen/Nikhef, Nijmegen, The Netherlands

${ }^{107}$ Nikhef National Institute for Subatomic Physics and University of Amsterdam, Amsterdam, The Netherlands

${ }^{108}$ Department of Physics, Northern Illinois University, De Kalb, IL, USA

${ }^{109}$ Budker Institute of Nuclear Physics, SB RAS, Novosibirsk, Russia

${ }^{110}$ Department of Physics, New York University, New York, NY, USA

111 Ohio State University, Columbus, OH, USA

${ }^{112}$ Faculty of Science, Okayama University, Okayama, Japan

${ }^{113}$ Homer L. Dodge Department of Physics and Astronomy, University of Oklahoma, Norman, OK, USA 
${ }^{114}$ Department of Physics, Oklahoma State University, Stillwater, OK, USA

115 Palacký University, RCPTM, Olomouc, Czech Republic

${ }^{116}$ Center for High Energy Physics, University of Oregon, Eugene, OR, USA

${ }^{117}$ LAL, Université Paris-Sud and CNRS/IN2P3, Orsay, France

118 Graduate School of Science, Osaka University, Osaka, Japan

${ }^{119}$ Department of Physics, University of Oslo, Oslo, Norway

${ }^{120}$ Department of Physics, Oxford University, Oxford, UK

121 (a) INFN Sezione di Pavia, Pavia, Italy; ${ }^{\text {(b) }}$ Dipartimento di Fisica, Università di Pavia, Pavia, Italy

${ }^{122}$ Department of Physics, University of Pennsylvania, Philadelphia, PA, USA

${ }^{123}$ National Research Centre "Kurchatov Institute" B.P. Konstantinov, Petersburg Nuclear Physics Institute, St. Petersburg, Russia

124 (a) INFN Sezione di Pisa, Pisa, Italy; ${ }^{(b)}$ Dipartimento di Fisica E. Fermi, Università di Pisa, Pisa, Italy

${ }^{125}$ Department of Physics and Astronomy, University of Pittsburgh, Pittsburgh, PA, USA

126 (a) Laboratório de Instrumentação e Física Experimental de Partículas-LIP, Lisbon, Portugal; ${ }^{(b)}$ Faculdade de Ciências, Universidade de Lisboa, Lisbon, Portugal; ${ }^{(c)}$ Department of Physics, University of Coimbra, Coimbra,

Portugal; ${ }^{(d)}$ Centro de Física Nuclear da Universidade de Lisboa, Lisbon, Portugal; ${ }^{(e)}$ Departamento de Fisica,

Universidade do Minho, Braga, Portugal; ${ }^{(\mathrm{f})}$ Departamento de Fisica Teorica y del Cosmos and CAFPE, Universidad de

Granada, Granada, Spain; ${ }^{(\mathrm{g})}$ Dep Fisica and CEFITEC of Faculdade de Ciencias e Tecnologia, Universidade Nova de

Lisboa, Caparica, Portugal

${ }^{127}$ Institute of Physics, Academy of Sciences of the Czech Republic, Prague, Czech Republic

${ }^{128}$ Czech Technical University in Prague, Prague, Czech Republic

${ }^{129}$ Faculty of Mathematics and Physics, Charles University in Prague, Prague, Czech Republic

${ }^{130}$ State Research Center Institute for High Energy Physics, Protvino, Russia

${ }^{131}$ Particle Physics Department, Rutherford Appleton Laboratory, Didcot, UK

132 (a) INFN Sezione di Roma, Rome, Italy; ${ }^{(\mathrm{b})}$ Dipartimento di Fisica, Sapienza Università di Roma, Rome, Italy

133 (a) INFN Sezione di Roma Tor Vergata, Rome, Italy; ${ }^{(b)}$ Dipartimento di Fisica, Università di Roma Tor Vergata, Rome, Italy

134 (a) INFN Sezione di Roma Tre, Rome, Italy; ${ }^{(b)}$ Dipartimento di Matematica e Fisica, Università Roma Tre, Rome, Italy

135 (a) Faculté des Sciences Ain Chock, Réseau Universitaire de Physique des Hautes Energies-Université Hassan II,

Casablanca, Morocco; ${ }^{\left({ }^{b}\right)}$ Centre National de l'Energie des Sciences Techniques Nucleaires, Rabat, Morocco; ${ }^{\left({ }^{c}\right)}$ Faculté

des Sciences Semlalia, Université Cadi Ayyad, LPHEA-Marrakech, Marrakech, Morocco; ${ }^{(d)}$ Faculté des Sciences,

Université Mohamed Premier and LPTPM, Oujda, Morocco; ${ }^{(e)}$ Faculté des Sciences, Université Mohammed V, Rabat,

Morocco

${ }^{136}$ DSM/IRFU (Institut de Recherches sur les Lois Fondamentales de l'Univers), CEA Saclay (Commissariat à l'Energie

Atomique et aux Energies Alternatives), Gif-sur-Yvette, France

${ }^{137}$ Santa Cruz Institute for Particle Physics, University of California Santa Cruz, Santa Cruz, CA, USA

${ }^{138}$ Department of Physics, University of Washington, Seattle, WA, USA

${ }^{139}$ Department of Physics and Astronomy, University of Sheffield, Sheffield, UK

${ }^{140}$ Department of Physics, Shinshu University, Nagano, Japan

${ }^{141}$ Fachbereich Physik, Universität Siegen, Siegen, Germany

142 Department of Physics, Simon Fraser University, Burnaby, BC, Canada

${ }^{143}$ SLAC National Accelerator Laboratory, Stanford, CA, USA

144 (a) Faculty of Mathematics, Physics and Informatics, Comenius University, Bratislava, Slovak Republic; (b) Department of Subnuclear Physics, Institute of Experimental Physics of the Slovak Academy of Sciences, Kosice, Slovak Republic

145 (a) Department of Physics, University of Cape Town, Cape Town, South Africa; ${ }^{(b)}$ Department of Physics, University of Johannesburg, Johannesburg, South Africa; ${ }^{(c)}$ School of Physics, University of the Witwatersrand, Johannesburg, South Africa

146 (a) Department of Physics, Stockholm University, Stockholm, Sweden; ${ }^{(b)}$ The Oskar Klein Centre, Stockholm, Sweden

${ }^{147}$ Physics Department, Royal Institute of Technology, Stockholm, Sweden

${ }^{148}$ Departments of Physics and Astronomy and Chemistry, Stony Brook University, Stony Brook, NY, USA

149 Department of Physics and Astronomy, University of Sussex, Brighton, UK

${ }^{150}$ School of Physics, University of Sydney, Sydney, Australia

${ }^{151}$ Institute of Physics, Academia Sinica, Taipei, Taiwan 
${ }^{152}$ Department of Physics, Technion: Israel Institute of Technology, Haifa, Israel

153 Raymond and Beverly Sackler School of Physics and Astronomy, Tel Aviv University, Tel Aviv, Israel

${ }^{154}$ Department of Physics, Aristotle University of Thessaloniki, Thessaloníki, Greece

155 International Center for Elementary Particle Physics and Department of Physics, The University of Tokyo, Tokyo, Japan

${ }^{156}$ Graduate School of Science and Technology, Tokyo Metropolitan University, Tokyo, Japan

${ }^{157}$ Department of Physics, Tokyo Institute of Technology, Tokyo, Japan

${ }^{158}$ Department of Physics, University of Toronto, Toronto, ON, Canada

159 (a) TRIUMF, Vancouver, BC, Canada; ${ }^{(b)}$ Department of Physics and Astronomy, York University, Toronto, ON, Canada

${ }^{160}$ Faculty of Pure and Applied Sciences, and Center for Integrated Research in Fundamental Science and Engineering,

University of Tsukuba, Tsukuba, Japan

${ }^{161}$ Department of Physics and Astronomy, Tufts University, Medford, MA, USA

${ }^{162}$ Centro de Investigaciones, Universidad Antonio Narino, Bogotá, Colombia

163 Department of Physics and Astronomy, University of California Irvine, Irvine, CA, USA

164 (a) INFN Gruppo Collegato di Udine, Sezione di Trieste, Udine, Italy; ${ }^{\left({ }^{b}\right)}$ ICTP, Trieste, Italy; ${ }^{(c)}$ Dipartimento di Chimica

Fisica e Ambiente, Università di Udine, Udine, Italy

165 Department of Physics, University of Illinois, Urbana, IL, USA

166 Department of Physics and Astronomy, University of Uppsala, Uppsala, Sweden

${ }^{167}$ Instituto de Física Corpuscular (IFIC) and Departamento de Física Atómica, Molecular y Nuclear and Departamento de Ingeniería Electrónica and Instituto de Microelectrónica de Barcelona (IMB-CNM), University of Valencia and CSIC, Valencia, Spain

168 Department of Physics, University of British Columbia, Vancouver, BC, Canada

${ }^{169}$ Department of Physics and Astronomy, University of Victoria, Victoria, BC, Canada

${ }^{170}$ Department of Physics, University of Warwick, Coventry, UK

${ }^{171}$ Waseda University, Tokyo, Japan

172 Department of Particle Physics, The Weizmann Institute of Science, Rehovot, Israel

173 Department of Physics, University of Wisconsin, Madison, WI, USA

${ }^{174}$ Fakultät für Physik und Astronomie, Julius-Maximilians-Universität, Würzburg, Germany

${ }^{175}$ Fachbereich C Physik, Bergische Universität Wuppertal, Wuppertal, Germany

176 Department of Physics, Yale University, New Haven, CT, USA

177 Yerevan Physics Institute, Yerevan, Armenia

${ }^{178}$ Centre de Calcul de l'Institut National de Physique Nucléaire et de Physique des Particules (IN2P3), Villeurbanne, France

a Also at Department of Physics, King's College London, London, UK

${ }^{\mathrm{b}}$ Also at Institute of Physics, Azerbaijan Academy of Sciences, Baku, Azerbaijan

${ }^{\mathrm{c}}$ Also at Novosibirsk State University, Novosibirsk, Russia

${ }^{\mathrm{d}}$ Also at TRIUMF, Vancouver, BC, Canada

e Also at Department of Physics, California State University, Fresno, CA, USA

${ }^{f}$ Also at Department of Physics, University of Fribourg, Fribourg, Switzerland

g Also at Departamento de Fisica e Astronomia, Faculdade de Ciencias, Universidade do Porto, Porto, Portugal

${ }^{\mathrm{h}}$ Also at Tomsk State University, Tomsk, Russia

${ }^{i}$ Also at CPPM, Aix-Marseille Université and CNRS/IN2P3, Marseille, France

j Also at Universita di Napoli Parthenope, Naples, Italy

${ }^{\mathrm{k}}$ Also at Institute of Particle Physics (IPP), Waterloo, Canada

${ }^{1}$ Also at Particle Physics Department, Rutherford Appleton Laboratory, Didcot, UK

${ }^{m}$ Also at Department of Physics, St. Petersburg State Polytechnical University, St. Petersburg, Russia

${ }^{n}$ Also at Louisiana Tech University, Ruston, LA, USA

${ }^{\circ}$ Also at Institucio Catalana de Recerca i Estudis Avancats, ICREA, Barcelona, Spain

p Also at Department of Physics, The University of Michigan, Ann Arbor MI, United States of America

q Also at Graduate School of Science, Osaka University, Osaka, Japan

${ }^{\mathrm{r}}$ Also at Department of Physics, National Tsing Hua University, Taiwan

${ }^{s}$ Also at Department of Physics, The University of Texas at Austin, Austin, TX, USA

${ }^{t}$ Also at Institute of Theoretical Physics, Ilia State University, Tbilisi, Georgia

u Also at CERN, Geneva, Switzerland 
${ }^{v}$ Also at Georgian Technical University (GTU), Tbilisi, Georgia

${ }^{w}$ Also at Manhattan College, New York, NY, USA

${ }^{x}$ Also at Hellenic Open University, Patras, Greece

y Also at Institute of Physics, Academia Sinica, Taipei, Taiwan

${ }^{\mathrm{z}}$ Also at LAL, Université Paris-Sud and CNRS/IN2P3, Orsay, France

aa Also at Academia Sinica Grid Computing, Institute of Physics, Academia Sinica, Taipei, Taiwan

${ }^{a b}$ Also at School of Physics, Shandong University, Shandong, China

${ }^{a c}$ Also at Moscow Institute of Physics and Technology State University, Dolgoprudny, Russia

${ }^{\text {ad }}$ Also at Section de Physique, Université de Genève, Geneva, Switzerland

${ }^{\text {ae }}$ Also at International School for Advanced Studies (SISSA), Trieste, Italy

af Also at Department of Physics and Astronomy, University of South Carolina, Columbia, SC, USA

ag Also at School of Physics and Engineering, Sun Yat-sen University, Guangzhou, China

ah Also at Faculty of Physics, M.V.Lomonosov Moscow State University, Moscow, Russia

ai Also at National Research Nuclear University MEPhI, Moscow, Russia

aj Also at Department of Physics, Stanford University, Stanford, CA, USA

ak Also at Institute for Particle and Nuclear Physics, Wigner Research Centre for Physics, Budapest, Hungary

${ }^{\text {al }}$ Also at University of Malaya, Department of Physics, Kuala Lumpur, Malaysia

* Deceased 